\title{
Diet and Nutrition Status of Mongolian Adults
}

\author{
Sabri Bromage 1,*(D), Tselmen Daria ${ }^{2}$, Rebecca L. Lander ${ }^{3}$, Soninkhishig Tsolmon ${ }^{4} \mathbb{D}_{\text {, }}$ \\ Lisa A. Houghton ${ }^{5}$, Enkhjargal Tserennadmid ${ }^{6}$, Nyamjargal Gombo ${ }^{7} \mathbb{D}$, Rosalind S. Gibson ${ }^{5}$ \\ and Davaasambuu Ganmaa ${ }^{8, *}$
}

1 Department of Nutrition, Harvard T.H. Chan School of Public Health, Boston, MA 02115, USA

2 Department of Neurology, Ulm University, Ulm 89081, Germany, and Central Scientific Laboratory, Institute of Medical Sciences, Ulaanbaatar 16081, Mongolia; tsemena312@gmail.com

3 Department of Pediatrics, Section of Nutrition, University of Colorado School of Medicine, Aurora, CO 80045, USA; rebecca.lander3@gmail.com

4 School of Public Health, Mongolian National University of Medical Sciences, Ulaanbaatar 14210, Mongolia; soninkhishig@must.edu.mn

5 Department of Human Nutrition, University of Otago, Dunedin 9054, New Zealand; lisa.houghton@otago.ac.nz (L.A.H.); rosalind.gibson@otago.ac.nz (R.S.G.)

6 Nutrition Laboratory, National Center for Public Health, Ulaanbaatar 13381, Mongolia; enke98@yahoo.com

7 Food and Agriculture Organization of the United Nations Country Office, Ulaanbaatar 14201, Mongolia; nyamjargal.gombo@fao.org

8 Department of Nutrition, Harvard T.H. Chan School of Public Health and Channing Division of Network Medicine, Department of Medicine, Brigham and Women's Hospital and Harvard Medical School, Boston, MA 02115, USA

* Correspondence: sbromage@mail.harvard.edu (S.B.); gdavaasa@hsph.harvard.edu (D.G.)

Received: 18 April 2020; Accepted: 19 May 2020; Published: 22 May 2020

check for updates

\begin{abstract}
Background: Aspects of the Mongolian food supply, including high availability of animal-source foods and few plant foods, are plausibly associated with disease in the population. Data on Mongolian diets are lacking, and these risks are poorly quantified. The purpose of this study was to provide a multifaceted nutritional analysis of the modern Mongolian diet. (2) Methods: The study population consisted of 167 male and 167 female healthy non-pregnant urban and nomadic adults (22-55 years) randomly selected from lists of residents in 8 regions. From 2011-2016, 3-day weighed diet records and serum were collected twice from each participant in summer and winter; anthropometry was collected once from each participant. Serum was analyzed for biomarkers, and nutrient intake computed using purpose-built food composition data and adjusted for within-person variation. Exploratory dietary patterns were derived and analyzed for associations with diet and nutrition measurements. (3) Results: We collected 1838 of an expected 1986 diet records (92.5\%), $610 / 658$ serum samples $(92.7 \%)$, and 315/334 height and weight measurements (94.3\%). Sixty-one percent of men and $51 \%$ of women were overweight or obese. Consumption of red meat, refined grains, and whole-fat dairy was high, while that of fruits, non-tuberous vegetables, eggs, nuts and seeds, fish and poultry, and whole grains was low. Dairy and red meat were more consumed in summer and winter, respectively. Dietary inadequacy of 10 of 21 assessed nutrients, including fiber, folate, and vitamin D were $>50 \%$ prevalent, while protein, zinc, and vitamin B12 inadequacy were low. Biochemical evidence of iron and vitamin A deficiency was also low. Three dietary patterns (Urban, Transitional, Nomadic) explained $41 \%$ of variation in food consumption. The Urban pattern was positively associated with BMI in multivariate analysis. (4) Conclusions: Results indicate a high prevalence of key dietary inadequacies and overweight among Mongolian adults. Prior studies by our group have suggested that expanded supplementation and food fortification would be effective in addressing micronutrient inadequacies; these strategies should be coupled with measures to mitigate the growing burden of chronic disease.
\end{abstract}


Keywords: nutrition assessment; diet survey; dietary pattern analysis; nutrient inadequacy; overweight and obesity; nutritional epidemiology; nutrition transition; pastoral nomadism; Mongolia; central Asia

\section{Introduction}

Due to climatic factors and a traditionally nomadic lifestyle, Mongolia contains a smaller fraction of arable land devoted to permanent crops than any other country $(0.003 \%)$ [1], and the national food supply is marked by a pattern of extremes. Normalized against the total per capita supply of caloric energy in each country, data from the Food and Agriculture Organization of the United Nations (FAO) from the most recent year available indicate that the daily per capita food supply of Mongolia ranked 9th and 12th out of 175 countries in terms of whole milk and meat supplied, respectively, and ranked in the bottom 10\% of countries in the supply of fruit, fish and seafood, pulses, and oil crops [2]. Particularly in rural areas, food consumption remains highly seasonal, especially with respect to dairy products and meat which are mostly consumed in summer and winter, respectively [3].

Extremes in Mongolia's national food supply are plausibly related to the national burden of disease, particularly chronic disease. Recent analysis of the Global Dietary Database shows that Mongolia scored lower than any other country in both 1990 and 2017 in the Alternative Healthy Eating Index [4]. Using FAO food balance data to estimate food consumption in Mongolia [5], the Global Burden of Disease study found that out of 195 countries and territories in 2017, Mongolia ranked 1st globally in the fraction of cardiovascular (CVD) mortality attributable to dietary imbalances in both men $(62.8 \%)$ and women $(60.5 \%)$ and 12 th in the rate of age-standardized all-cause mortality attributable to diet (309 deaths per 100,000) [6]. Mongolia also ranked 22nd and 12th in rates of all-cause and CVD mortality attributable to metabolic risk factors. While childhood stunting and vitamin A deficiency have decreased over the past two decades, this period has also seen a sharp rise in the prevalence of adolescent and adult overweight and type 2 diabetes [7,8]. Currently, almost one third of pregnant women are anemic, less than two thirds of infants are reportedly exclusively breastfed up to six months of age, and vitamin D deficiency is endemic [7,9].

Despite these concerning statistics, national assessments of dietary intakes of individuals are lacking for the Mongolian population. From 1999-2009, dietary assessments of individuals and analytic epidemiologic research on Mongolian and Inner Mongolian diets were pioneered by Mongolian and Japanese scientists [10-15], but methods used in these studies were not applied on a nationwide scale. Current nationally-representative food or nutrient consumption data the individual level are not available. Without such data, it is impossible to precisely quantify the extent of dietary imbalances throughout the population, and difficult to design evidence-based and targeted strategies to address such imbalances.

The objective of this study was to provide a multifaceted nutritional analysis of the modern Mongolian diet, using paired summer and winter weighed diet records collected from urban and rural Mongolian men and women living in 8 regions of the country. As part of a prior analysis based on these diet records [16], we reported the extent of population dietary inadequacy of 10 micronutrients; the present analysis extends this investigation to 21 nutrients, includes analysis of daily intake of food groups and their contributions to nutrient intakes, and includes exploratory analysis of population diet patterns. To support interpretation of the dietary data, we also assessed anthropometry and selected micronutrient biomarkers.

\section{Materials and Methods}

\subsection{Study Population and Data Collection}

The study population consisted of 167 male and 167 female healthy non-pregnant adults aged 22-55 and living in separate households. Eligible participants were randomly sampled from lists 
of urban and peri-urban residents of the capital Ulaanbaatar and the provincial centers of 7 aimags (provinces) spanning the country (Bulgan, Dornod, Khuvsgul, Khovd, Omnogobi, Sukhbaatar, and Tuv), and nomads residing in soums (rural districts) within a short drive from each provincial center (Archon, Bayantumen, Chagall, Buyant, Bayandalai, Khalzan, and Altanbulag, respectively). Participants were located and followed-up with the aid of local health officers (Figure 1).

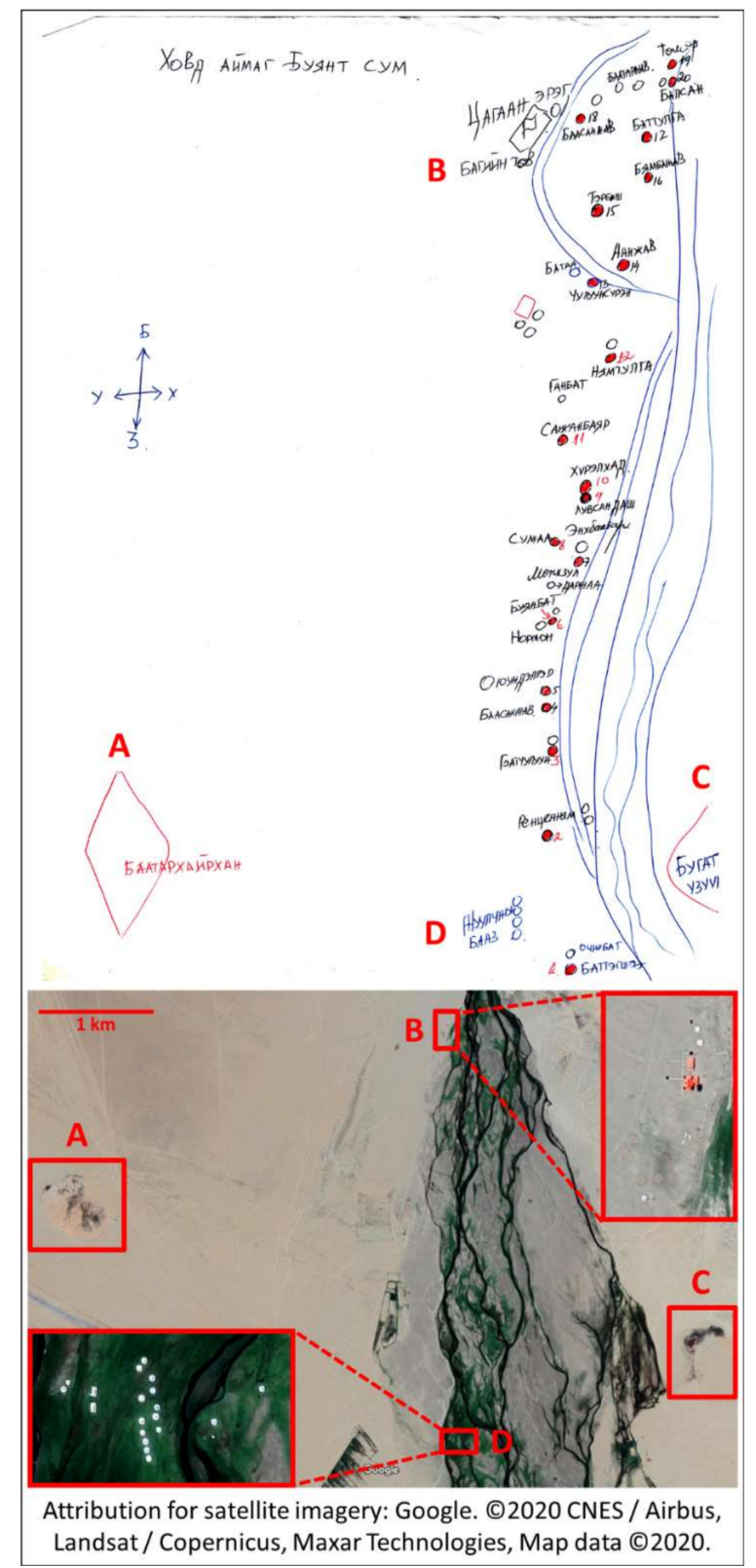

Figure 1. A health officer's map of rural participant locations juxtaposed with satellite imagery. In rural areas, local health department staff are skilled at locating and tracking surrounding nomadic families, despite their frequent movements. This is exemplified by this map, quickly drafted from memory for the study team's use by a local health office working in the rural Buyant subdistrict of Khovd province (western Mongolia). The following landmarks can be found in the map and the satellite map of the same study site: A: "Baatarkhairkhan" mountain; B: local administration center, C: "Deer antler" rock formation, D: tourist camp. Attribution for satellite imagery: Google Maps. (C2020 CNES / Airbus, Landsat / Copernicus, Maxar Technologies, Map data @2020. https://www.google.com/maps/@47. 9632414,91.593478,7154a,35y,217.12h/data=!3m1!1e3. 
From 2011 to 2016, 3 consecutive days of weighed diet records (including 1 weekend day, and including assessment of portions consumed as well as ingredient composition of mixed dishes), were collected from each participant by dedicated research assistants in both summer (June-August) and winter (December-February). In addition, serum samples were collected from each participant during both seasons, and height and weight measured in the winter only for the majority of participants. As fasting is not required for accurate measurement of the biomarkers of interest, serum was collected at random times during the day in order to avoid systematic error in dietary measurements that could occur from interrupting all subjects at the same time (e.g., before breakfast). Details of the dietary assessment, blood collection, and anthropometry have been described earlier $[9,17]$.

Procedures followed were in accordance with the ethical standards of the Mongolian Ministry of Health Ethical Review Board and the Harvard T.H. Chan School of Public Health Institutional Review Board (Protocol 21002). Eligible participants provided written informed consent prior to enrolment and were free to withdraw from the study at any time.

\subsection{Analysis of Anthropometric and Micronutrient Status}

Body mass index was calculated using participants' height and weight, and categorized according to WHO cutoffs [18] based on evidence suggesting that these are more appropriate than WPRO Asian-specific cutoffs for use in Mongolia [19]. Serum was analyzed for ferritin, soluble transferrin receptor (sTfR), retinol binding protein (RBP), standard C-reactive protein (CRP, a marker of acute-phase systemic inflammation), and alpha-1-acid glycoprotein (AGP, a marker of chronic inflammation) using a validated sandwich ELISA [20]. Iron and vitamin A deficiency were defined according to serum ferritin and RBP concentration cutoffs, respectively, differentially adjusted for inflammatory defined according to different combinations of elevated CRP $(5 \mathrm{mg} / \mathrm{L})$ and AGP $(1 \mathrm{~g} / \mathrm{L})$ : normal-, incubation/late convalescence-, and early convalescence-specific cutoffs used for ferritin and RBP were 15/19/27 $\mu \mathrm{g} / \mathrm{L}$ and $0.7 / 0.6 / 0.5 \mu \mathrm{mol} / \mathrm{L}$, respectively [21]. Iron overload was defined as serum ferritin $>300 \mathrm{ng} / \mathrm{mL}$ in men and $>200 \mathrm{ng} / \mathrm{mL}$ in women without the presence of inflammation [22].

Means and prevalence of categories of BMI and biomarkers were calculated within subgroups (defined by urbanicity and sex) and season, as well as within regions in both seasons. Significant differences $(p<0.05)$ were identified for the following planned comparisons: summer vs. winter within urbanicity-sex, males vs. females within urbanicity (BMI) or urbanicity-season (serum biomarkers), urban vs. rural within sex (BMI) or sex-season, and each province vs. the mean of all other provinces. Least squares analysis was conducted to identify marginal effects of urbanicity, sex, and age on mean biomarkers, adjusting for covariates. Statistical analyses were performed in $R$ version 3.4.3 ( $R$ Foundation for Statistical Computing, Vienna, Austria).

\subsection{Analysis of Diet Records}

Empirical recipes and dish yields [23] were generated for complex dishes by averaging information on the weight of raw ingredients versus cooked dishes when available, or by applying ingredient yield factors [24,25]. Nutrient composition of ingredients, single-ingredient food items, and complex dishes were compiled using a combination of unpublished locally-analyzed food composition data from the Mongolian University of Science and Technology and Mongolian Public Health Institute, food composition data from the United States and Germany [26,27], and entries from a combination of international food composition tables previously compiled as part of a food composition table for Mongolian children [28]. Where applicable, nutrient retention factors [24,29,30] were applied to calculate nutrient concentrations in cooked foods, and adjustments made, where necessary, to borrowed nutrient values for differences between the moisture and fat content of the Mongolian and borrowed foods [23].

Usual intake distributions of nutrients and nutritionally-relevant food groups were estimated for each of the 8 subgroup-seasons (e.g., Urban Males in Summer) and each of the 8 regions, using the Statistical Program to Assess Dietary Exposure (SPADE) in R [31], which corrects observed 
measurements for within-person variation using variance components estimated from the study population. For each food group or nutrient, the estimation method used in each subgroup-season was dependent on the observed frequency of consumption as follows (Table S1):

(1) Distribution of habitually-consumed components (those observed to be consumed on at least $95 \%$ of diet record days, including some food groups and all nutrients except alcohol) was estimated using a model that estimates intake amounts only.

(2) Distribution of episodically-consumed components (those consumed on less than 95\% of diet record days, including alcohol and most food groups) was estimated using a model that estimates both intake frequencies and intake amounts.

(3) In cases where consumption of a food group was too infrequent for the episodic model to produce an intake distribution, mean intake was calculated by computing each person's average daily intake in each season and then averaging within-person seasonal means across persons in each subgroup-season.

Estimated distributions were used to derive mean intake of foods and nutrients, prevalence of nutrient inadequacy, and prevalence of intakes above the upper limit for all participants in each stratum as well as by tertiles of age $(<33,33-44,45+$ years $)$. Statistics were weighted according to the distribution of weekdays and weekend days. A full-probability approach was used to estimate inadequacy for all nutrients whose requirement was assumed to be normally distributed with a coefficient of variance $(\mathrm{CV})$ of $10 \%$ except for protein $(\mathrm{CV}=12.5 \%)$, vitamin $\mathrm{A}(20 \%)$, and niacin and copper (15\%) [32]; for iron among women of reproductive age, a lognormal requirement distribution was assumed. Given the population's high observed meat consumption and low phytate:zinc molar ratio, EARs from the U.S. National Academy of Medicine were considered appropriate [33].

The following quantities were also calculated in each stratum using the sequential averaging procedure described in (3) above:

(1) For each food group, mean daily intake density (g/2500 kcal/day, where 2500 kcal was approximately equal to the grand mean daily energy intake in the study population) was calculated to aid comparison of food intake across strata with varying levels of energy intake.

(2) A separate set of "dish-based" food groups (distinguished by local culinary practices instead of inherent nutritional significance) were analyzed for their mean proportional contributions to total daily intake of each nutrient.

(3) The mean fractional contribution of protein $(4 \mathrm{kcal} / \mathrm{g})$, carbohydrate $(4 \mathrm{kcal} / \mathrm{g})$, fat $(9 \mathrm{kcal} / \mathrm{g})$, and alcohol $(7 \mathrm{kcal} / \mathrm{g})$ to total energy intake; mean fraction of vitamin A contributed by retinol; and phytate:calcium, phytate:iron, and phytate:zinc molar ratios were calculated.

Exploratory diet patterns were analyzed using the "prcomp" package in R. Patterns were generated based on summer and winter within-person daily means of 12 food groups selected as major contributors of energy and nutrient intake in the study population. Patterns were retained according to quantitative criteria (variance explained by each rotated factor, and all factors as a whole, based on their associated eigenvalues and inspection of a scree plot) and qualitative criteria (interpretability of each factor with respect to its combination of high and low factor loadings, and the distribution of adherence to each pattern across population subgroups). Factor loadings were used to calculate season-specific patterns scores for each participant, and scaled from 0-100. Estimated marginal means of diet pattern scores were compared by age group, and selected nutrient intakes and biomarker measurements were compared across quintiles of diet pattern scores.

\section{Results}

Based on a possible maximum of 6 diet records (3 per season), 2 serum samples (1 per season), and 1 height and weight measurement collected from each participant, participants (167 men and 167 women) completed 1838 of 1986 (92.5\%) diet records (mean number of records per person: 5.50), 
provided 610 of $658(92.7 \%)$ serum samples (mean number of samples per person: 1.83), and 315 of 334 (94.3\%) height and weight measurements (Table S2). Mean age of participants was 39.2 years (Table S3). Additional population characteristics have been reported previously [9]. There were no significant differences in age, sex, urbanicity, province, or season between participants who provided a serum sample in both summer and winter and 3.9\% who provided only one sample.

Urban men and women consumed more fruits and non-tuberous vegetables than their rural counterparts in both seasons, with urban women consuming markedly more fruit than any other population subgroup (62 and $69 \mathrm{~g} /$ day in summer and winter, respectively) (Table 1). However, consumption of fruits, non-tuberous vegetables, eggs, nuts and seeds, fish and poultry, and whole grains was generally low across population subgroups and seasons, and consumption of deep orange tubers was not observed. Total consumption of milk and dairy products ranged from $116 \mathrm{~g} /$ day among urban males in winter to $657 \mathrm{~g} /$ day among rural males in summer, with rural men and women consuming more than their urban counterparts in both seasons, and summer consumption exceeding that in winter among all subgroups. Consumption of reduced-fat milk or dairy products was not observed. Consumption of juice and sugar-sweetened beverages (SSBs) was highest in urban areas, particularly among urban males (178 and $138 \mathrm{~g}$ /day in summer and winter, respectively), while consumption of sweets was highest among urban females (28 and $34 \mathrm{~g} /$ day in summer and winter, respectively), and approximately double that of their rural counterparts. Consumption of meat was extremely high, particularly among men and in winter (with urban and rural men consuming 425 and $450 \mathrm{~g} /$ day in winter, respectively), as was that of refined grains (ranging from $322 \mathrm{~g} /$ day among rural females in summer and $533 \mathrm{~g}$ /day among rural males in winter). Age-trends in food consumption are presented in Table S4.

Across subgroups and seasons, protein, carbohydrates, fat, and alcohol contributed an average of $21 \%, 40 \%, 38 \%$, and $1 \%$ of caloric energy, respectively, and mean phytate intake did not exceed 500 $\mathrm{mg}$ (Table 2). The prevalence of dietary protein, copper, phosphorous, zinc, riboflavin, niacin, and vitamin B12 inadequacy was very low, only exceeding $10 \%$ for riboflavin and niacin among urban females in winter. A moderate prevalence of iron inadequacy was observed among urban women (19 and $21 \%$ in summer and winter, respectively) and rural women (16 and $14 \%)$. Inadequacies of calcium, magnesium, thiamin, and vitamins $\mathrm{A}$ and $\mathrm{B} 6$ were common, and almost the entire study population was inadequate in folate and vitamins C, D, and E in both seasons. Median intakes of dietary fiber and potassium fell short of sex-specific adequate intake levels, and median intakes of manganese and pantothenic acid generally met or exceeded adequate levels (median intake (IQR) of fiber, potassium, manganese, and pantothenic acid averaged across the 8 subgroup-seasons: 9.3 (4.3), 2732 (1117), 3.176 (1.568), 6.354 (2.596), respectively). Urban-rural differences in nutrient inadequacy were most salient for calcium (more commonly inadequate in urban areas), vitamin A (more common in urban areas in summer, and rural areas in winter), and vitamin B6 (more common in rural areas in summer). The prevalence of intakes above the upper limit did not exceed $10 \%$ except for calcium among rural males in summer (12\%) (Table S5), due to their high summer intake of milk and dairy products (mean: 657 $\mathrm{g} /$ day) which contributed $62 \%$ of their total calcium intake in that season. Age-trends in nutrient intake and adequacy are presented in Table S6.

Dishes contributing the majority of dietary energy in urban and rural areas included bread with or without toppings (contributing an average of $10 \%$ across urban and rural men and women in summer and winter, and $8 \%$ across rural subgroups), milk, dairy products, and airag (fermented mares' milk) (10 and $18 \%$ in urban and rural subgroups respectively), biscuits, cookies, and doughnuts (12 and $18 \%$ ), stir fries (21 and 15\%), soups (14 and 13\%), dumplings not included in soup (14 and 13\%), and miscellaneous meat dishes ( 9 and 10\%) (Table 3). See Table S7 for each dish's contribution to other nutrients. 
Table 1. Mean usual intake (g/day) and intake density (g/2500 kcal/day) of food groups by subgroup-season and province.

\begin{tabular}{|c|c|c|c|c|c|c|c|c|c|c|c|c|c|c|c|c|c|}
\hline \multirow[t]{2}{*}{$\overline{7}$} & \multirow[t]{2}{*}{ Food Group } & $\begin{array}{l}\text { Urban } \\
\text { Male } \\
\text { Summer }\end{array}$ & $\begin{array}{l}\text { Urban } \\
\text { Male } \\
\text { Winter }\end{array}$ & $\begin{array}{l}\text { Urban } \\
\text { Female } \\
\text { Summer }\end{array}$ & $\begin{array}{l}\text { Urban } \\
\text { Female } \\
\text { Winter }\end{array}$ & $\begin{array}{c}\text { Rural } \\
\text { Male } \\
\text { Summer }\end{array}$ & $\begin{array}{l}\text { Rural } \\
\text { Male } \\
\text { Winter }\end{array}$ & $\begin{array}{c}\text { Rural } \\
\text { Female } \\
\text { Summer }\end{array}$ & $\begin{array}{l}\text { Rural } \\
\text { Female } \\
\text { Winter }\end{array}$ & Ulaanbaatar & Tuv & Buglan & Khuvsgul & Khovd & Omnogobi & Sukhbaatar & Dornod \\
\hline & & $269 \mathrm{DR}$ & $\begin{array}{l}230 \\
\text { DR }\end{array}$ & 278 DR & $\begin{array}{l}246 \\
\text { DR }\end{array}$ & $216 \mathrm{DR}$ & $\begin{array}{l}200 \\
\text { DR }\end{array}$ & 206 DR & $\begin{array}{l}193 \\
\text { DR }\end{array}$ & $219 \mathrm{DR}$ & $\begin{array}{l}227 \\
\text { DR }\end{array}$ & $\begin{array}{l}239 \\
\text { DR }\end{array}$ & $231 \mathrm{DR}$ & $\begin{array}{l}228 \\
\text { DR }\end{array}$ & $241 \mathrm{DR}$ & $227 \mathrm{DR}$ & $226 \mathrm{DR}$ \\
\hline \multirow{31}{*}{ 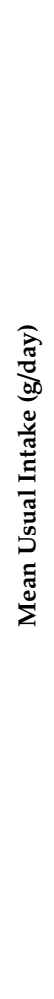 } & Fruit & 15.5 & 16.1 & 61.5 & 69.0 & 9.3 & 7.5 & 13.5 & 12.5 & 21.4 & 13.1 & 32.8 & 13.0 & 31.3 & 30.8 & 13.8 & 29.8 \\
\hline & Citrus fruits & 0.0 & 1.0 & 0.0 & 0.1 & 0.0 & 0.0 & 0.0 & 0.0 & 0.0 & 0.0 & 0.0 & 0.0 & 0.0 & 0.0 & 1.1 & 0.0 \\
\hline & Deep orange fruits & 3.4 & 0.4 & 3.9 & 1.3 & 2.4 & 0.2 & 0.0 & 0.4 & 1.8 & 2.8 & 1.6 & 1.0 & 0.2 & 0.4 & 0.7 & 5.3 \\
\hline & Other fruits & 12.1 & 14.7 & 57.6 & 67.5 & 7.0 & 7.3 & 13.5 & 12.1 & 19.6 & 10.3 & 31.2 & 11.9 & 31.1 & 30.4 & 12.0 & 24.6 \\
\hline & Non-Tuberous Vegetables & 64.1 & 61.6 & 61.2 & 46.3 & 50.1 & 41.6 & 26.2 & 28.6 & 64.3 & 55.2 & 42.4 & 46.6 & 35.8 & 49.5 & 41.0 & 54.6 \\
\hline & Cruciferous vegetables & 14.4 & 10.8 & 13.0 & 11.4 & 13.0 & 7.2 & 3.1 & 5.8 & 15.0 & 9.1 & 12.7 & 7.8 & 8.1 & 9.9 & 9.4 & 9.2 \\
\hline & Dark green leafy vegetables & 0.0 & 0.3 & 0.7 & 0.1 & 0.2 & 0.0 & 0.2 & 0.0 & 0.5 & 0.0 & 0.1 & 0.0 & 0.0 & 0.4 & 0.1 & 0.6 \\
\hline & Deep orange vegetables & 0.6 & 0.0 & 0.0 & 0.0 & 0.0 & 0.0 & 0.0 & 0.0 & 0.0 & 0.0 & 0.0 & 0.0 & 0.6 & 0.1 & 0.0 & 0.0 \\
\hline & Legumes & 0.4 & 1.9 & 0.2 & 0.5 & 0.0 & 0.0 & 0.0 & 0.0 & 0.0 & 0.6 & 0.0 & 0.5 & 0.0 & 0.0 & 1.9 & 0.0 \\
\hline & Other vegetables & 48.8 & 48.5 & 47.3 & 34.2 & 36.8 & 34.5 & 22.8 & 22.8 & 48.7 & 45.5 & 29.6 & 38.3 & 27.2 & 39.1 & 29.6 & 44.7 \\
\hline & Dairy & 153.4 & 115.8 & 163.6 & 127.2 & 656.5 & 256.7 & 373.9 & 181.9 & 129.0 & 533.4 & 199.4 & 255.9 & 214.6 & 219.1 & 246.3 & 260.6 \\
\hline & Milk (incl. airag) & 103.3 & 78.5 & 93.6 & 80.4 & 578.7 & 231.3 & 288.8 & 157.6 & 75.4 & 487.7 & 162.7 & 182.2 & 149.1 & 165.9 & 199.3 & 203.6 \\
\hline & Dairy products & 50.2 & 37.3 & 70.0 & 46.7 & 77.8 & 25.3 & 85.1 & 24.3 & 53.6 & 45.7 & 36.7 & 73.7 & 65.5 & 53.2 & 47.0 & 57.0 \\
\hline & Eggs & 13.1 & 10.3 & 12.0 & 8.4 & 2.6 & 0.6 & 2.5 & 1.3 & 17.3 & 4.4 & 9.9 & 5.2 & 2.4 & 8.0 & 3.5 & 6.3 \\
\hline & Juice and SSBs & 177.8 & 138.7 & 65.3 & 54.8 & 41.4 & 17.7 & 39.2 & 12.4 & 130.2 & 89.1 & 43.6 & 104.4 & 75.4 & 92.0 & 48.7 & 50.2 \\
\hline & Juice & 57.2 & 47.2 & 36.8 & 39.8 & 18.1 & 10.7 & 17.7 & 5.0 & 36.2 & 32.6 & 15.0 & 55.5 & 43.1 & 17.9 & 25.8 & 27.0 \\
\hline & Sugar-sweetened beverages & 120.6 & 91.5 & 28.5 & 14.9 & 23.3 & 7.1 & 21.5 & 7.3 & 94.0 & 56.5 & 28.7 & 48.9 & 32.3 & 74.1 & 22.9 & 23.1 \\
\hline & Liquid Oils & 8.0 & 5.0 & 7.2 & 4.0 & 4.8 & 3.2 & 3.9 & 4.0 & 6.4 & 4.7 & 3.1 & 4.2 & 4.2 & 4.1 & 4.5 & 4.8 \\
\hline & Nuts and Seeds & 1.2 & 0.0 & 2.5 & 0.5 & 1.0 & 0.0 & 1.6 & 0.0 & 0.4 & 1.8 & 0.7 & 0.2 & 0.9 & 2.9 & 1.0 & 0.5 \\
\hline & Sweets & 13.3 & 12.3 & 27.8 & 34.2 & 10.5 & 12.8 & 15.3 & 16.5 & 21.4 & 15.5 & 16.2 & 17.6 & 18.9 & 25.9 & 19.8 & 17.2 \\
\hline & Red and Processed Meat & 354.8 & 424.7 & 244.3 & 240.0 & 302.3 & 450.1 & 205.6 & 295.1 & 343.4 & 322.5 & 296.9 & 317.0 & 341.6 & 299.0 & 302.8 & 291.3 \\
\hline & Processed meat & 15.6 & 14.0 & 4.7 & 3.9 & 2.6 & 4.9 & 6.1 & 0.9 & 23.1 & 6.3 & 2.3 & 9.3 & 4.6 & 2.6 & 2.8 & 7.3 \\
\hline & Red meat & 339.3 & 410.7 & 239.6 & 236.1 & 299.8 & 445.2 & 199.5 & 294.2 & 320.2 & 316.2 & 294.6 & 307.8 & 337.0 & 296.4 & 300.1 & 284.0 \\
\hline & Lean Meat & 5.6 & 5.2 & 5.1 & 1.5 & 0.2 & 13.3 & 1.3 & 6.3 & 4.4 & 6.8 & 0.6 & 1.6 & 1.7 & 15.9 & 4.5 & 1.2 \\
\hline & Fish & 1.7 & 0.7 & 0.2 & 1.5 & 0.2 & 12.8 & 1.3 & 6.3 & 0.7 & 1.6 & 0.0 & 1.6 & 0.6 & 15.3 & 0.8 & 0.8 \\
\hline & Poultry & 3.9 & 4.5 & 4.9 & 0.0 & 0.0 & 0.5 & 0.0 & 0.0 & 3.8 & 5.2 & 0.6 & 0.0 & 1.1 & 0.6 & 3.7 & 0.4 \\
\hline & Grains & 457.9 & 474.1 & 399.9 & 333.5 & 413.5 & 532.8 & 322.1 & 368.7 & 432.3 & 397.2 & 396.5 & 442.2 & 466.9 & 369.0 & 380.6 & 397.1 \\
\hline & Refined grains & 452.7 & 465.4 & 392.9 & 322.0 & 409.6 & 531.7 & 314.6 & 365.8 & 428.8 & 392.7 & 394.9 & 438.1 & 461.3 & 364.1 & 360.9 & 394.9 \\
\hline & Whole grains & 5.2 & 8.7 & 7.0 & 11.5 & 3.9 & 1.2 & 7.4 & 2.9 & 3.4 & 4.5 & 1.6 & 4.0 & 5.5 & 4.9 & 19.8 & 2.2 \\
\hline & $\begin{array}{l}\text { Deep Orange Roots and } \\
\text { Tubers }\end{array}$ & 0.0 & 0.0 & 0.0 & 0.0 & 0.0 & 0.0 & 0.0 & 0.0 & 0.0 & 0.0 & 0.0 & 0.0 & 0.0 & 0.0 & 0.0 & 0.0 \\
\hline & White Roots and Tubers & 77.6 & 75.2 & 49.0 & 56.9 & 60.3 & 52.4 & 41.2 & 36.0 & 82.6 & 61.2 & 53.7 & 50.9 & 49.7 & 53.4 & 49.1 & 59.0 \\
\hline
\end{tabular}


Table 1. Cont.

\begin{tabular}{|c|c|c|c|c|c|c|c|c|c|c|c|c|c|c|c|c|c|}
\hline \multirow[t]{2}{*}{$\overline{7}$} & \multirow[t]{2}{*}{ Food Group } & $\begin{array}{l}\text { Urban } \\
\text { Male } \\
\text { Summer }\end{array}$ & $\begin{array}{l}\text { Urban } \\
\text { Male } \\
\text { Winter }\end{array}$ & $\begin{array}{l}\text { Urban } \\
\text { Female } \\
\text { Summer }\end{array}$ & $\begin{array}{l}\text { Urban } \\
\text { Female } \\
\text { Winter }\end{array}$ & $\begin{array}{c}\text { Rural } \\
\text { Male } \\
\text { Summer }\end{array}$ & $\begin{array}{l}\text { Rural } \\
\text { Male } \\
\text { Winter }\end{array}$ & $\begin{array}{c}\text { Rural } \\
\text { Female } \\
\text { Summer }\end{array}$ & $\begin{array}{l}\text { Rural } \\
\text { Female } \\
\text { Winter }\end{array}$ & Ulaanbaatar & Tuv & Buglan & Khuvsgul & Khovd & Omnogobi & Sukhbaatar & Dornod \\
\hline & & 269 DR & $\begin{array}{l}230 \\
\text { DR }\end{array}$ & 278 DR & $\begin{array}{l}246 \\
\text { DR }\end{array}$ & 216 DR & $\begin{array}{l}200 \\
\text { DR }\end{array}$ & 206 DR & $\begin{array}{l}193 \\
\text { DR }\end{array}$ & 219 DR & $\begin{array}{l}227 \\
\text { DR }\end{array}$ & $\begin{array}{l}239 \\
\text { DR }\end{array}$ & 231 DR & $\begin{array}{l}228 \\
\text { DR }\end{array}$ & $241 \mathrm{DR}$ & 227 DR & $226 \mathrm{DR}$ \\
\hline \multirow{31}{*}{ 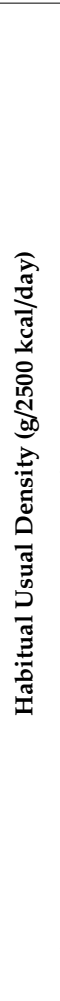 } & Fruit & 17.4 & 14.1 & 60.6 & 53.1 & 9.4 & 7.7 & 13.8 & 12.9 & 22.7 & 12.1 & 45.4 & 14.9 & 28.4 & 31.5 & 15.7 & 33.2 \\
\hline & Citrus fruits & 0.0 & 0.9 & 0.0 & 0.1 & 0.0 & 0.0 & 0.0 & 0.0 & 0.0 & 0.0 & 0.0 & 0.0 & 0.0 & 0.0 & 1.2 & 0.0 \\
\hline & Deep orange fruits & 3.0 & 0.3 & 4.6 & 1.3 & 2.0 & 0.2 & 0.0 & 0.5 & 1.7 & 2.4 & 1.7 & 1.0 & 0.1 & 0.6 & 0.9 & 5.8 \\
\hline & Other fruits & 14.4 & 12.8 & 56.0 & 51.6 & 7.4 & 7.5 & 13.8 & 12.4 & 21.0 & 9.7 & 43.7 & 13.9 & 28.3 & 30.9 & 13.6 & 27.4 \\
\hline & Non-tuberous vegetables & 60.6 & 56.2 & 75.0 & 61.8 & 43.3 & 32.9 & 35.6 & 32.4 & 63.5 & 56.3 & 44.3 & 46.4 & 32.9 & 58.8 & 43.6 & 61.2 \\
\hline & Cruciferous vegetables & 14.1 & 10.1 & 12.9 & 13.8 & 9.1 & 6.6 & 6.8 & 6.4 & 15.4 & 8.6 & 13.5 & 7.8 & 7.9 & 10.5 & 9.9 & 9.8 \\
\hline & Dark green leafy vegetables & 0.0 & 0.3 & 1.0 & 0.2 & 0.3 & 0.0 & 0.3 & 0.0 & 0.5 & 0.0 & 0.1 & 0.0 & 0.0 & 0.6 & 0.1 & 0.7 \\
\hline & Deep orange vegetables & 0.5 & 0.0 & 0.0 & 0.0 & 0.0 & 0.0 & 0.0 & 0.0 & 0.0 & 0.0 & 0.0 & 0.0 & 0.6 & 0.1 & 0.0 & 0.0 \\
\hline & Legumes & 0.3 & 2.0 & 0.2 & 0.7 & 0.0 & 0.0 & 0.0 & 0.0 & 0.0 & 0.6 & 0.0 & 0.6 & 0.0 & 0.0 & 2.2 & 0.0 \\
\hline & Other vegetables & 45.7 & 43.8 & 61.0 & 47.0 & 33.9 & 26.3 & 28.4 & 26.0 & 47.5 & 47.1 & 30.6 & 38.0 & 24.5 & 47.5 & 31.4 & 50.7 \\
\hline & Dairy & 175.1 & 100.5 & 205.9 & 156.8 & 606.6 & 210.7 & 461.2 & 213.6 & 138.5 & 497.5 & 219.5 & 231.5 & 202.7 & 243.9 & 245.7 & 290.1 \\
\hline & Milk (incl. airag) & 130.2 & 79.8 & 126.4 & 101.9 & 533.8 & 191.2 & 355.3 & 187.5 & 76.9 & 453.0 & 175.8 & 157.8 & 144.0 & 186.8 & 205.9 & 226.3 \\
\hline & Dairy products & 44.9 & 20.7 & 79.5 & 54.9 & 72.8 & 19.4 & 105.9 & 26.0 & 61.6 & 44.5 & 43.7 & 73.7 & 58.7 & 57.1 & 39.8 & 63.8 \\
\hline & Eggs & 12.4 & 10.4 & 13.8 & 12.8 & 2.5 & 0.8 & 2.3 & 1.5 & 15.0 & 4.3 & 12.5 & 5.1 & 3.3 & 9.0 & 4.8 & 7.4 \\
\hline & Juice and SSBs & 188.1 & 114.4 & 84.9 & 68.3 & 35.1 & 12.2 & 41.0 & 13.8 & 121.1 & 78.5 & 51.4 & 106.5 & 59.1 & 94.0 & 57.8 & 53.9 \\
\hline & Juice & 42.7 & 48.9 & 53.6 & 49.7 & 11.8 & 7.1 & 13.6 & 5.7 & 39.5 & 29.6 & 18.8 & 58.4 & 39.7 & 19.3 & 32.0 & 26.2 \\
\hline & Sugar-sweetened beverages & 145.4 & 65.6 & 31.3 & 18.6 & 23.3 & 5.2 & 27.4 & 8.1 & 81.6 & 48.9 & 32.6 & 48.2 & 19.4 & 74.8 & 25.9 & 27.7 \\
\hline & Liquid Oils & 6.0 & 4.5 & 4.7 & 3.8 & 4.4 & 3.9 & 3.9 & 3.9 & 6.2 & 4.3 & 3.5 & 3.9 & 3.8 & 4.2 & 4.5 & 5.0 \\
\hline & Nuts and Seeds & 0.7 & 0.0 & 2.5 & 0.4 & 0.6 & 0.0 & 1.6 & 0.0 & 0.2 & 1.3 & 0.6 & 0.2 & 0.8 & 2.7 & 0.9 & 0.6 \\
\hline & Sweets & 15.8 & 13.8 & 31.5 & 32.3 & 9.6 & 8.6 & 20.5 & 19.7 & 22.5 & 16.1 & 19.7 & 17.8 & 16.9 & 24.3 & 20.1 & 18.9 \\
\hline & Red and Processed Meat & 324.4 & 376.1 & 260.4 & 303.0 & 286.3 & 359.7 & 250.2 & 342.3 & 310.7 & 296.4 & 321.0 & 297.4 & 295.2 & 317.6 & 315.7 & 295.2 \\
\hline & Processed meat & 12.7 & 12.7 & 5.2 & 5.9 & 2.8 & 2.9 & 8.6 & 1.0 & 18.9 & 6.0 & 2.4 & 8.7 & 4.1 & 3.4 & 3.4 & 8.5 \\
\hline & Red meat & 311.7 & 363.4 & 255.2 & 297.1 & 283.5 & 356.9 & 241.5 & 341.3 & 291.8 & 290.4 & 318.6 & 288.6 & 291.1 & 314.2 & 312.3 & 286.7 \\
\hline & Lean Meat & 3.7 & 6.0 & 5.2 & 1.9 & 0.3 & 9.8 & 1.6 & 5.9 & 4.7 & 6.4 & 0.4 & 1.8 & 2.4 & 14.7 & 4.7 & 1.4 \\
\hline & Fish & 1.7 & 0.7 & 0.4 & 1.9 & 0.3 & 9.4 & 1.6 & 5.9 & 0.7 & 1.8 & 0.0 & 1.8 & 1.6 & 14.0 & 1.1 & 0.9 \\
\hline & Poultry & 2.1 & 5.3 & 4.7 & 0.0 & 0.0 & 0.3 & 0.0 & 0.0 & 4.0 & 4.6 & 0.4 & 0.0 & 0.9 & 0.6 & 3.7 & 0.5 \\
\hline & Grains & 400.3 & 421.2 & 425.0 & 407.4 & 372.1 & 407.3 & 381.0 & 419.7 & 412.0 & 376.0 & 404.9 & 448.3 & 410.2 & 379.5 & 400.7 & 408.5 \\
\hline & Refined grains & 395.2 & 417.5 & 415.8 & 398.1 & 368.0 & 406.2 & 373.7 & 415.8 & 408.8 & 371.9 & 402.7 & 444.4 & 405.5 & 375.3 & 380.1 & 406.4 \\
\hline & Whole grains & 5.1 & 3.7 & 9.3 & 9.3 & 4.1 & 1.1 & 7.3 & 3.8 & 3.2 & 4.1 & 2.3 & 3.9 & 4.6 & 4.2 & 20.6 & 2.0 \\
\hline & $\begin{array}{l}\text { Deep Orange Roots and } \\
\text { Tubers }\end{array}$ & 0.0 & 0.0 & 0.0 & 0.0 & 0.0 & 0.0 & 0.0 & 0.0 & 0.0 & 0.0 & 0.0 & 0.0 & 0.0 & 0.0 & 0.0 & 0.0 \\
\hline & White Roots and Tubers & 74.0 & 67.4 & 55.8 & 70.7 & 55.1 & 43.3 & 50.2 & 40.6 & 78.2 & 56.2 & 58.2 & 49.6 & 46.7 & 56.7 & 52.7 & 61.7 \\
\hline
\end{tabular}
The total number of diet records (DR) available for analysis are listed below each column heading. Depending on data availability, usual intakes within subgroup-seasons are either
estimated using habitual or episodic intake models analyzing up to 3 days per person per season, or calculated by first computing each person's average daily intake in each season and then averaging these within-person seasonal means across persons in each subgroup-season (intake densities are only calculated using this sequential averaging procedure). Usual intakes and intake densities within provinces are calculated by first averaging each person's summer and winter daily means and then averaging these within-person annual means across persons in each province. To aid interpretation, intake densities are expressed on a per $2500 \mathrm{kcal} /$ day basis, which is approximately equal to the mean daily energy intake in the study population. Intake models are estimated using SPADE [31]. Statistics are weighted by weekday of each record day. Airag: fermented mares' milk, SSBs: sugar-sweetened beverages. 
Table 2. Mean usual nutrient intake (per day) and prevalence of nutrient inadequacy ( $\%<$ estimated average requirement) by subgroup-season and province.

\begin{tabular}{|c|c|c|c|c|c|c|c|c|c|c|c|c|c|c|c|c|c|}
\hline \multirow[t]{2}{*}{ 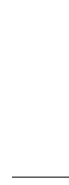 } & \multirow[t]{2}{*}{ Nutrient } & $\begin{array}{l}\text { Urban } \\
\text { Male } \\
\text { Summer }\end{array}$ & $\begin{array}{l}\text { Urban } \\
\text { Male } \\
\text { Winter }\end{array}$ & $\begin{array}{l}\text { Urban } \\
\text { Female } \\
\text { Summer }\end{array}$ & $\begin{array}{l}\text { Urban } \\
\text { Female } \\
\text { Winter }\end{array}$ & $\begin{array}{l}\text { Rural } \\
\text { Male } \\
\text { Summer }\end{array}$ & $\begin{array}{l}\text { Rural } \\
\text { Male } \\
\text { Winter }\end{array}$ & $\begin{array}{l}\text { Rural } \\
\text { Female } \\
\text { Summer }\end{array}$ & $\begin{array}{l}\text { Rural } \\
\text { Female } \\
\text { Winter }\end{array}$ & Ulaanbaatar & r Tuv & Buglan & Khuvsgul & Khovd & Omnogobi & Sukhbaatar & Dornod \\
\hline & & $269 \mathrm{DR}$ & $\begin{array}{l}230 \\
\text { DR }\end{array}$ & 278 DR & $\begin{array}{l}246 \\
\text { DR }\end{array}$ & 216 DR & $\begin{array}{l}200 \\
\text { DR }\end{array}$ & 206 DR & $\begin{array}{l}193 \\
\text { DR }\end{array}$ & 219 DR & $\begin{array}{l}227 \\
\text { DR }\end{array}$ & $\begin{array}{l}239 \\
\text { DR }\end{array}$ & 231 DR & $\begin{array}{l}228 \\
\text { DR }\end{array}$ & $241 \mathrm{DR}$ & 227 DR & $226 \mathrm{DR}$ \\
\hline \multirow{35}{*}{ 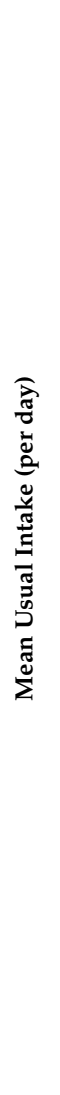 } & Energy (kcal) & 2972 & 2897 & 2339 & 2069 & 2752 & 3266 & 2113 & 2214 & 2603 & 2664 & 2359 & 2634 & 2857 & 2407 & 2441 & 2478 \\
\hline & Protein (g) & 160.96 & 161.56 & 113.10 & 101.98 & 145.41 & 171.62 & 103.99 & 115.10 & 136.30 & 149.76 & 119.60 & 128.01 & 139.26 & 130.71 & 132.66 & 124.68 \\
\hline & per $\mathrm{kg}$ & 2.1 & 2.1 & 1.8 & 1.6 & 1.9 & 2.3 & 1.7 & 1.9 & 2.0 & 2.1 & 1.5 & 1.8 & 2.1 & 2.0 & 1.9 & 1.8 \\
\hline & $\%$ total energy & 21.3 & 22.4 & 19.1 & 20.1 & 21.0 & 21.5 & 19.9 & 21.4 & 20.9 & 21.6 & 20.4 & 19.3 & 19.5 & 21.8 & 21.4 & 20.4 \\
\hline & Available carbohydrates (g) & 284.73 & 267.29 & 240.22 & 211.99 & 256.70 & 295.44 & 208.60 & 213.22 & 253.82 & 250.57 & 227.59 & 258.22 & 267.06 & 230.48 & 237.75 & 245.51 \\
\hline & $\%$ total energy & 40.8 & 38.8 & 43.8 & 42.4 & 37.8 & 37.4 & 40.2 & 39.5 & 41.2 & 38.9 & 39.8 & 41.8 & 38.9 & 40.0 & 40.9 & 41.6 \\
\hline & Total fat $(\mathrm{g})$ & 121.15 & 120.32 & 97.58 & 85.47 & 117.75 & 144.80 & 92.97 & 96.28 & 109.79 & 111.51 & 103.89 & 111.30 & 128.98 & 99.32 & 98.11 & 102.98 \\
\hline & $\%$ total energy & 36.0 & 37.4 & 36.6 & 37.0 & 37.5 & 39.8 & 39.2 & 38.9 & 37.6 & 36.1 & 38.9 & 37.1 & 41.0 & 36.9 & 36.5 & 36.9 \\
\hline & Alcohol, (g) & 6.86 & 7.00 & 1.66 & 0.94 & 14.98 & 5.15 & 2.66 & 0.37 & 1.15 & 15.91 & 2.68 & 7.38 & 3.13 & 6.02 & 4.65 & 4.97 \\
\hline & $\%$ total energy & 2.0 & 1.4 & 0.5 & 0.4 & 3.7 & 1.2 & 0.7 & 0.2 & 0.3 & 3.4 & 0.8 & 1.8 & 0.6 & 1.3 & 1.2 & 1.0 \\
\hline & Fiber (g) & 11.7 & 10.9 & 11.0 & 9.1 & 9.3 & 10.7 & 7.2 & 7.9 & 11.1 & 9.7 & 8.8 & 9.7 & 10.6 & 8.9 & 9.1 & 10.3 \\
\hline & Phytosterols (mg) & 455 & 431 & 386 & 333 & 416 & 500 & 328 & 353 & 409 & 397 & 384 & 365 & 440 & 388 & 386 & 408 \\
\hline & Calcium (mg) & 663 & 518 & 594 & 461 & 1361 & 730 & 852 & 515 & 541 & 1092 & 573 & 709 & 626 & 640 & 683 & 757 \\
\hline & Phytate:Ca molar ratio & 0.1 & 0.1 & 0.1 & 0.1 & 0.0 & 0.1 & 0.0 & 0.1 & 0.1 & 0.0 & 0.1 & 0.0 & 0.1 & 0.1 & 0.0 & 0.0 \\
\hline & Copper (mg) & 1.933 & 1.932 & 1.731 & 1.322 & 2.642 & 2.078 & 2.101 & 1.405 & 1.723 & 1.777 & 1.603 & 1.872 & 2.060 & 1.860 & 2.013 & 2.056 \\
\hline & Iron (mg) & 20.61 & 20.86 & 15.49 & 13.48 & 18.98 & 23.38 & 14.52 & 16.18 & 17.77 & 18.45 & 15.02 & 18.84 & 19.84 & 16.99 & 17.72 & 17.79 \\
\hline & Phytate:Fe molar ratio & 2.1 & 1.9 & 2.5 & 2.3 & 2.3 & 2.0 & 2.4 & 2.0 & 2.3 & 2.1 & 2.4 & 1.9 & 2.1 & 2.5 & 2.3 & 2.3 \\
\hline & Magnesium (mg) & 345 & 367 & 277 & 251 & 351 & 389 & 244 & 277 & 316 & 367 & 284 & 323 & 329 & 274 & 302 & 309 \\
\hline & Manganese (mg) & 4.170 & 4.054 & 3.266 & 2.663 & 3.166 & 4.056 & 2.411 & 2.966 & 3.584 & 3.345 & 3.163 & 3.526 & 3.818 & 2.967 & 3.054 & 3.200 \\
\hline & Phosphorus (mg) & 1501 & 1519 & 1145 & 1042 & 1649 & 1864 & 1251 & 1260 & 1303 & 1497 & 1203 & 1376 & 1591 & 1355 & 1363 & 1392 \\
\hline & Potassium (mg) & 3234 & 3478 & 2473 & 2335 & 2996 & 3538 & 2166 & 2457 & 2892 & 3153 & 2532 & 2789 & 2948 & 2670 & 2788 & 2724 \\
\hline & Zinc (mg) & 22.32 & 24.79 & 16.95 & 15.23 & 20.50 & 25.37 & 14.61 & 16.56 & 21.05 & 19.99 & 18.13 & 19.70 & 21.16 & 19.60 & 18.43 & 18.13 \\
\hline & Phytate:Zn molar ratio & 2.1 & 2.0 & 2.5 & 2.5 & 2.3 & 2.3 & 2.5 & 2.4 & 2.2 & 2.3 & 2.3 & 2.1 & 2.4 & 2.4 & 2.4 & 2.6 \\
\hline & Vitamin A (ug retinol eq.) & 577 & 559 & 628 & 413 & 1008 & 490 & 808 & 308 & 526 & 514 & 507 & 653 & 611 & 551 & 632 & 680 \\
\hline & $\%$ retinol & 51.8 & 54.8 & 63.0 & 59.4 & 73.6 & 67.3 & 80.6 & 68.5 & 55.4 & 61.5 & 59.1 & 64.4 & 66.7 & 67.5 & 70.1 & 69.7 \\
\hline & Thiamin (mg) & 1.259 & 1.112 & 1.027 & 0.810 & 1.414 & 1.156 & 0.970 & 0.811 & 1.096 & 1.203 & 0.839 & 1.230 & 1.125 & 0.970 & 0.980 & 1.203 \\
\hline & Riboflavin (mg) & 2.178 & 2.040 & 1.801 & 1.397 & 2.672 & 2.358 & 2.225 & 1.560 & 1.827 & 1.846 & 1.564 & 2.173 & 2.261 & 2.010 & 2.157 & 2.197 \\
\hline & Niacin (mg) & 27.406 & 27.237 & 19.954 & 16.250 & 24.086 & 27.311 & 17.022 & 17.575 & 23.778 & 22.445 & 18.710 & 23.653 & 23.452 & 22.323 & 21.217 & 21.622 \\
\hline & Pantothenic acid (mg) & 7.569 & 7.480 & 5.605 & 4.998 & 7.638 & 8.093 & 5.812 & 5.641 & 6.568 & 6.637 & 5.583 & 6.472 & 7.318 & 6.346 & 7.020 & 6.660 \\
\hline & Vitamin B6 (mg) & 1.332 & 1.480 & 0.920 & 0.917 & 1.018 & 1.394 & 0.786 & 0.980 & 1.156 & 1.138 & 0.939 & 1.195 & 1.050 & 1.026 & 1.103 & 1.119 \\
\hline & Folate (ug DFE) & 166 & 163 & 143 & 119 & 170 & 191 & 128 & 135 & 142 & 149 & 133 & 162 & 219 & 140 & 127 & 138 \\
\hline & Vitamin B12 (ug) & 13.18 & 12.22 & 8.31 & 6.86 & 17.64 & 12.96 & 15.15 & 8.79 & 10.81 & 10.70 & 9.60 & 10.27 & 13.28 & 12.21 & 13.87 & 11.66 \\
\hline & Vitamin C (mg) & 35.3 & 42.2 & 33.2 & 38.0 & 25.6 & 22.7 & 17.6 & 13.4 & 38.3 & 28.0 & 21.0 & 31.7 & 31.8 & 26.5 & 21.8 & 24.4 \\
\hline & Vitamin D (IU) & 39 & 31 & 34 & 27 & 45 & 43 & 36 & 25 & 40 & 37 & 31 & 35 & 27 & 43 & 36 & 36 \\
\hline & Vitamin E (mg) & 8.31 & 8.62 & 6.88 & 5.60 & 6.86 & 9.25 & 5.49 & 6.73 & 7.92 & 7.36 & 6.77 & 7.08 & 8.94 & 6.65 & 6.02 & 6.98 \\
\hline
\end{tabular}


Table 2. Cont.

\begin{tabular}{|c|c|c|c|c|c|c|c|c|c|c|c|c|c|c|c|c|c|}
\hline & \multirow[t]{2}{*}{ Nutrient } & $\begin{array}{l}\text { Urban } \\
\text { Male } \\
\text { Summer }\end{array}$ & $\begin{array}{l}\text { Urban } \\
\text { Male } \\
\text { Winter }\end{array}$ & $\begin{array}{c}\text { Urban } \\
\text { Female } \\
\text { Summer }\end{array}$ & $\begin{array}{l}\text { Urban } \\
\text { Female } \\
\text { Winter }\end{array}$ & $\begin{array}{c}\text { Rural } \\
\text { Male } \\
\text { Summer }\end{array}$ & $\begin{array}{c}\text { Rural } \\
\text { Male } \\
\text { Winter }\end{array}$ & $\begin{array}{c}\text { Rural } \\
\text { Female } \\
\text { Summer }\end{array}$ & $\begin{array}{l}\text { Rural } \\
\text { Female } \\
\text { Winter }\end{array}$ & Ulaanbaatar & Tuv & Buglan & Khuvsgul & Khovd & Omnogobi & Sukhbaatar & Dornod \\
\hline & & $269 \mathrm{DR}$ & $\begin{array}{l}230 \\
\text { DR }\end{array}$ & 278 DR & $\begin{array}{l}246 \\
\text { DR }\end{array}$ & $216 \mathrm{DR}$ & $\begin{array}{l}200 \\
\text { DR }\end{array}$ & $206 \mathrm{DR}$ & $\begin{array}{l}193 \\
\text { DR }\end{array}$ & $219 \mathrm{DR}$ & $\begin{array}{l}227 \\
\text { DR }\end{array}$ & $\begin{array}{l}239 \\
\text { DR }\end{array}$ & 231 DR & $\begin{array}{l}228 \\
\text { DR }\end{array}$ & $241 \mathrm{DR}$ & 227 DR & $226 \mathrm{DR}$ \\
\hline \multirow{17}{*}{ 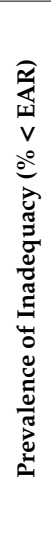 } & Protein (per kg) & 0.0 & 0.2 & 2.6 & 3.0 & 0.1 & 0.0 & 0.6 & 0.2 & 0.0 & 0.0 & 0.4 & 0.0 & 0.1 & 0.2 & 0.3 & 0.3 \\
\hline & Calcium & 74.1 & 94.6 & 85.4 & 95.3 & 31.8 & 66.7 & 55.5 & 88.9 & 93.7 & 40.8 & 88.7 & 71.5 & 95.2 & 78.5 & 72.6 & 65.8 \\
\hline & Copper & 0.1 & 0.0 & 0.3 & 2.8 & 0.1 & 0.0 & 0.1 & 0.8 & 0.1 & 0.0 & 0.2 & 0.0 & 0.0 & 0.0 & 0.0 & 0.0 \\
\hline & Iron & 0.0 & 0.0 & 19.0 & 21.4 & 0.1 & 0.0 & 15.7 & 14.1 & 7.5 & 7.4 & 7.5 & 6.6 & 7.3 & 8.9 & 7.4 & 6.9 \\
\hline & Magnesium & 51.6 & 47.6 & 48.5 & 60.1 & 55.5 & 33.4 & 64.6 & 47.9 & 48.1 & 33.6 & 64.6 & 42.3 & 43.6 & 66.2 & 54.8 & 54.5 \\
\hline & Phosphorus & 0.0 & 0.1 & 5.0 & 9.6 & 0.4 & 0.0 & 0.2 & 1.0 & 1.1 & 0.1 & 1.7 & 0.1 & 0.0 & 0.7 & 0.6 & 0.6 \\
\hline & Zinc & 0.1 & 0.2 & 1.7 & 0.2 & 0.4 & 0.1 & 0.2 & 0.8 & 0.6 & 0.3 & 1.3 & 0.0 & 0.2 & 0.4 & 0.0 & 2.1 \\
\hline & Vitamin A & 65.8 & 68.9 & 48.5 & 73.5 & 41.8 & 75.9 & 20.4 & 89.7 & 65.5 & 66.5 & 66.9 & 41.3 & 45.6 & 61.0 & 51.0 & 29.1 \\
\hline & Thiamin & 28.9 & 43.8 & 46.5 & 67.0 & 27.1 & 35.8 & 44.3 & 67.5 & 45.0 & 26.5 & 69.7 & 28.0 & 20.3 & 52.0 & 49.3 & 35.1 \\
\hline & Riboflavin & 2.5 & 0.7 & 3.8 & 13.5 & 3.7 & 0.7 & 0.3 & 4.1 & 4.7 & 0.0 & 7.9 & 0.5 & 0.0 & 1.3 & 1.6 & 2.2 \\
\hline & Niacin & 0.2 & 0.6 & 8.1 & 12.6 & 2.5 & 0.3 & 5.5 & 8.6 & 5.0 & 1.9 & 11.1 & 0.7 & 1.5 & 1.7 & 0.2 & 8.4 \\
\hline & Vitamin B6 & 35.7 & 27.9 & 74.1 & 77.4 & 67.5 & 23.5 & 90.7 & 72.1 & 53.1 & 54.8 & 86.8 & 52.1 & 62.8 & 65.3 & 59.8 & 58.1 \\
\hline & Folate & 98.7 & 97.9 & 96.4 & 98.9 & 95.8 & 93.0 & 99.9 & 98.3 & 99.3 & 99.5 & 99.7 & 98.1 & 89.6 & 99.2 & 100.0 & 98.6 \\
\hline & Vitamin B12 & 0.0 & 0.0 & 0.0 & 0.0 & 0.0 & 0.0 & 0.0 & 0.0 & 0.0 & 0.0 & 0.0 & 0.0 & 0.0 & 0.0 & 0.0 & 0.0 \\
\hline & Vitamin C & 98.9 & 98.7 & 96.1 & 87.4 & 99.7 & 100.0 & 100.0 & 100.0 & 100.0 & 98.0 & 100.0 & 99.0 & 98.1 & 98.3 & 100.0 & 99.9 \\
\hline & Vitamin D & 100.0 & 100.0 & 100.0 & 100.0 & 100.0 & 100.0 & 100.0 & 100.0 & 100.0 & 100.0 & 100.0 & 100.0 & 100.0 & 100.0 & 100.0 & 100.0 \\
\hline & Vitamin E & 95.3 & 89.0 & 94.1 & 98.2 & 95.7 & 88.7 & 100.0 & 93.9 & 95.7 & 97.6 & 97.8 & 98.1 & 90.9 & 96.6 & 99.8 & 94.6 \\
\hline
\end{tabular}

The total number of diet records (DR) available for analysis are listed below each column heading. Within subgroup-seasons and provinces, usual intakes of all nutrients (except alcohol) and nutrient adequacy prevalence are estimated using habitual intake models analyzing up to 3 or 6 record days per person, respectively, while nutrient proportions and ratios are calculated using the sequential averaging procedures described in the footnote to Table 1. Depending on data availability, usual alcohol intake is estimated either using episodic intake estimated using SPADE [31]. Statistics are weighted by weekday of each record day. Shading is proportional to nutrient inadequacy prevalence (white: $0.0 \%$, red: $100.0 \%$ ). 
Table 3. Mean percentage of dietary energy contributed by consumed dishes by subgroup-season and province.

\begin{tabular}{|c|c|c|c|c|c|c|c|c|}
\hline \multirow[t]{2}{*}{ Food Group } & $\begin{array}{l}\text { Urban Male } \\
\text { Summer }\end{array}$ & $\begin{array}{l}\text { Urban Male } \\
\text { Winter }\end{array}$ & $\begin{array}{l}\text { Urban Female } \\
\text { Summer }\end{array}$ & $\begin{array}{c}\text { Urban Female } \\
\text { Winter }\end{array}$ & $\begin{array}{l}\text { Rural Male } \\
\text { Summer }\end{array}$ & $\begin{array}{c}\text { Rural Male } \\
\text { Winter }\end{array}$ & $\begin{array}{l}\text { Rural Female } \\
\text { Summer }\end{array}$ & $\begin{array}{c}\text { Rural Female } \\
\text { Winter }\end{array}$ \\
\hline & 269 DR & $230 \mathrm{DR}$ & 278 DR & 246 DR & 216 DR & $200 \mathrm{DR}$ & $206 \mathrm{DR}$ & 193 DR \\
\hline Airag & 0.9 & 0.1 & 0.3 & 0.0 & 4.5 & 0.2 & 1.5 & 0.3 \\
\hline Beer, wine, spirits & 2.8 & 2.3 & 0.8 & 0.7 & 4.4 & 2.6 & 0.4 & 0.2 \\
\hline Biscuits, cookies, doughnuts & 8.1 & 10.1 & 13.2 & 16.4 & 12.3 & 20.6 & 19.1 & 20.7 \\
\hline Bread with or without toppings & 10.5 & 8.2 & 11.8 & 9.5 & 11.2 & 4.5 & 11.5 & 4.7 \\
\hline Dairy products (excl. milk), eggs & 3.8 & 2.3 & 6.3 & 5.7 & 4.9 & 3.1 & 7.7 & 4.4 \\
\hline Dumplings (excl. in soup) & 11.7 & 19.3 & 10.7 & 15.9 & 7.6 & 20.5 & 5.7 & 19.2 \\
\hline Juice, SSBs & 3.4 & 2.1 & 1.9 & 1.5 & 0.7 & 0.3 & 0.8 & 0.2 \\
\hline Milk (excl. airag), milk tea & 4.1 & 4.5 & 5.6 & 5.4 & 12.0 & 8.5 & 13.6 & 9.9 \\
\hline Miscellaneous foods & 1.6 & 1.3 & 1.2 & 1.7 & 0.8 & 0.8 & 0.7 & 1.2 \\
\hline Nutrient-dense snacks & 0.6 & 0.6 & 3.2 & 1.9 & 0.3 & 0.3 & 0.7 & 0.4 \\
\hline Other meat dishes & 8.6 & 12.1 & 5.9 & 7.5 & 9.5 & 10.7 & 8.8 & 9.1 \\
\hline Soups & 16.6 & 14.0 & 13.5 & 11.2 & 13.0 & 12.5 & 11.8 & 12.8 \\
\hline Stir fries & 24.8 & 21.0 & 20.0 & 17.8 & 18.2 & 14.0 & 15.2 & 14.0 \\
\hline Sweets, ice cream & 1.2 & 1.2 & 3.8 & 3.4 & 0.6 & 1.0 & 2.2 & 2.5 \\
\hline \multirow[t]{3}{*}{ Tea, coffee, water } & 1.1 & 0.9 & 1.7 & 1.5 & 0.1 & 0.6 & 0.1 & 0.5 \\
\hline & Ulaanbaatar & Tuv & Bulgan & Khuvsgul & Khovd & Omnogobi & Sukhbaatar & Dornod \\
\hline & 219 DR & 227 DR & $239 \mathrm{DR}$ & $231 \mathrm{DR}$ & 228 DR & $241 \mathrm{DR}$ & 227 DR & 226 DR \\
\hline Airag & 0.1 & 4.7 & 1.6 & 0.3 & 0.0 & 1.0 & 0.0 & 0.1 \\
\hline Beer, wine, spirits & 0.6 & 4.0 & 0.7 & 2.7 & 1.1 & 1.3 & 2.3 & 1.9 \\
\hline Biscuits, cookies, doughnuts & 9.2 & 12.3 & 19.1 & 12.5 & 23.2 & 16.2 & 11.6 & 12.4 \\
\hline Bread with or without toppings & 11.2 & 8.1 & 4.9 & 12.8 & 7.1 & 6.8 & 10.4 & 13.2 \\
\hline Dairy products (excl. milk), eggs & 5.6 & 4.9 & 4.5 & 4.3 & 6.0 & 5.7 & 2.7 & 4.8 \\
\hline Dumplings (excl. in soup) & 10.2 & 13.5 & 15.8 & 15.5 & 12.7 & 12.5 & 14.2 & 11.1 \\
\hline Juice, SSBs & 2.3 & 1.4 & 1.0 & 2.1 & 1.4 & 1.9 & 1.1 & 1.1 \\
\hline Milk (excl. airag), milk tea & 4.1 & 6.8 & 4.9 & 6.0 & 6.7 & 8.5 & 13.3 & 11.0 \\
\hline Miscellaneous foods & 1.7 & 1.4 & 0.7 & 1.6 & 0.7 & 0.7 & 1.0 & 1.6 \\
\hline Nutrient-dense snacks & 0.7 & 0.9 & 1.5 & 0.8 & 0.9 & 1.7 & 1.2 & 1.2 \\
\hline Other meat dishes & 9.1 & 7.7 & 8.0 & 6.9 & 9.3 & 12.4 & 8.7 & 8.5 \\
\hline Soups & 16.0 & 13.6 & 14.2 & 15.3 & 12.1 & 10.6 & 14.6 & 11.7 \\
\hline Stir fries & 25.7 & 18.0 & 19.3 & 16.2 & 16.0 & 17.6 & 15.2 & 19.3 \\
\hline Sweets, ice cream & 1.7 & 1.9 & 2.3 & 1.9 & 1.9 & 2.4 & 2.2 & 1.7 \\
\hline Tea, coffee, water & 1.9 & 0.6 & 1.3 & 1.1 & 1.1 & 0.6 & 1.5 & 0.4 \\
\hline
\end{tabular}

The total number of diet records (DR) available for analysis are listed in each column heading. Within subgroup-seasons and provinces, means are calculated using the sequential averaging procedures described in the footnote to Table 1. Shading is proportional to mean percentage contribution (white: $0.0 \%$, blue: $25.7 \%$ ). Airag: fermented mares' milk, SSBs: sugar-sweetened beverages. See Table $\mathrm{S} 7$ for a brief description of each food group. 
Of the study population, $61 \%$ of the men and $51 \%$ of the women were overweight, with $16 \%$ and $8 \%$, respectively, being obese; $3 \%$ were underweight (Table 4). Mean BMI was significantly higher among men than women in both urban areas (men: $26.9 \mathrm{~kg} / \mathrm{m}^{2}$, women: 25.5 ) and rural areas (men: 26.0, women: 24.6), and did not differ across urban and rural areas within sex. In adjusted analyses, urban residence was independently associated with higher BMI (adjusted mean: 26.4 vs. 24.9 in urban and rural areas, respectively) (Table 5). Biochemical iron deficiency, iron overload, and vitamin A deficiency were observed only in $n=12,4$, and 2 samples, respectively, and elevated CRP and AGP concentrations were observed in 11 and $7 \%$ of samples, respectively (Table 4 ). Urban residence was independently associated with higher serum RBP and lower AGP concentrations (Table 5).

Factor loadings, and the mean observed intake of each factor component across quintiles of factor scores in both seasons combined are presented in Table 6 and Figure 2. Three patterns were retained: an "Urban" pattern (attributing 21\% of variance in intake of factor components) marked by high consumption of vegetables, juice and sugar-sweetened beverages, liquid oils, red meat, refined grains, and white roots and tubers; a "Transitional" pattern (11\% of variance) marked by high consumption of dairy products, sweets, and fruit, and low alcohol and red meat; and a "Nomadic" pattern $(10 \%$ of variance) marked by high consumption of dairy products, milk, red meat, and refined grains, and low juice and SSBs, processed meat, and fruit. In adjusted analyses, adherence to the Urban pattern was significantly higher among urban and Ulaanbaatar residents; the Transitional pattern was most associated with female sex, and the Nomadic pattern with rural residence (Table 7). Urban pattern scores were independently associated with younger age in all subgroups except rural females, the Transitional pattern was not associated with age in any subgroup, and Nomadic pattern scores increased with age among urban and rural males (Table 8, Figure 3). Adjusting for total energy intake and other covariates, increased adherence to the Urban pattern was significantly associated with increased intakes of protein, fiber, iron, and zinc, and decreased calcium intake; the Transitional pattern with increased protein intake; and both the Transitional and Nomadic patterns with increased intakes of iron and zinc, and decreased fiber intake (Table 9). 
Table 4. Mean body mass index and serum biomarker concentrations by subgroup, subgroup-season, and province.

\begin{tabular}{|c|c|c|c|c|c|c|c|c|c|c|c|c|c|c|c|c|}
\hline \multirow{3}{*}{ Measurement } & \multicolumn{4}{|c|}{$\begin{array}{l}\text { Urban Male } 93 \text { Participants) } \\
\text { (a) }\end{array}$} & \multicolumn{4}{|c|}{ Urban Female (96 Participants) } & \multicolumn{4}{|c|}{ Rural Male (74 Participants) } & \multirow{2}{*}{\multicolumn{4}{|c|}{ Rural Female (71 Participants) }} \\
\hline & \multicolumn{2}{|c|}{ Mean or $n$} & \multirow{2}{*}{\multicolumn{2}{|c|}{ SE or $\%$}} & \multicolumn{2}{|c|}{ Mean or $n$} & \multirow{2}{*}{\multicolumn{2}{|c|}{ SE or \% }} & \multicolumn{2}{|c|}{ Mean or $n$} & \multirow{2}{*}{\multicolumn{2}{|c|}{ SE or $\%$}} & & & & \\
\hline & \multirow{3}{*}{\multicolumn{2}{|c|}{$\begin{array}{c}26.9 \\
1\end{array}$}} & \multirow{2}{*}{\multicolumn{2}{|c|}{$\begin{array}{l}0.4 \\
1.1 \%\end{array}$}} & \multirow{2}{*}{\multicolumn{2}{|c|}{$\begin{array}{c}25.5 \\
3\end{array}$}} & & & \multirow{2}{*}{\multicolumn{2}{|c|}{$\begin{array}{c}26.0 \\
0\end{array}$}} & & & \multicolumn{2}{|c|}{$\begin{array}{l}\text { Mean or } n \\
24.6\end{array}$} & \multicolumn{2}{|c|}{$\begin{array}{c}S E \text { or } \% \\
0.5\end{array}$} \\
\hline Underweight $(<18.5)$ & & & & & & & \multicolumn{2}{|c|}{$\begin{array}{l}0.4 \\
3.1 \%\end{array}$} & & & \multicolumn{2}{|c|}{$0.0 \%$} & \multirow{2}{*}{\multicolumn{2}{|c|}{$\begin{array}{c}1 \\
39\end{array}$}} & \multicolumn{2}{|c|}{$1.4 \%$} \\
\hline Normal (18.5-25) & & & $31.2>3$. & & 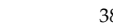 & & 39.6 & & 3: 5 & & & & & & & \\
\hline Overweight (25-30) & & & 50.5 - & & $4: 3$ & & 46.9 & & 2 & & $39.2>37>3$ & & 2 & & & \\
\hline Obese $(30+)$ & & & 17.2 & & $1 \mathrm{c}$ & & 10.4 & & 1 & & 13. & & & & & \\
\hline & Urban Ma & Summer & Urban Ma & Winter & $\begin{array}{r}\text { Urban I } \\
\text { Sum }\end{array}$ & $\begin{array}{l}\text { emale } \\
\text { ter }\end{array}$ & Urban Fem & le winter & Rural Mal & Summer & Rural Ma & Winter & $\begin{array}{r}\text { Rural I } \\
\text { Sum }\end{array}$ & $\begin{array}{l}\text { emale } \\
\text { ner }\end{array}$ & Rural Fem & le Winter \\
\hline & 90 Meast & ements & 83 Measu & ements & 93 Measu & ements & 87 Measu & ements & 72 Measu & rements & 72 Measu & ements & 70 Meast & ements & 68 Meast & rements \\
\hline & Mean or $n$ & SE or $\%$ & Mean or $n$ & SE or $\%$ & Mean or $n$ & SE or $\%$ & Mean or $n$ & SE or $\%$ & Mean or $n$ & SE or $\%$ & Mean or $n$ & SE or $\%$ & Mean or $n$ & SE or $\%$ & Mean or $n$ & SE or $\%$ \\
\hline Mean ferritin (ug/L) & 162.1 & 4.7 & 157.7 & 4.8 & 93.5 & 4.7 & 90.8 & 5.5 & 153.2 & 5.4 & 139.7 & 5.5 & 106.3 & 5.4 & 106.7 & 5.5 \\
\hline Iron deficient $* *$ & 0 & $0.0 \%$ & 0 & $0.0 \%$ & 2 & $2.2 \%$ & 5 & $5.9 \%$ & 0 & $0.0 \%$ & 0 & $0.0 \%$ & 4 & $5.9 \%$ & 1 & $1.6 \%$ \\
\hline Iron overload ** & 0 & $0.0 \%$ & 0 & $0.0 \%$ & 1 & $1.1 \%$ & 1 & $1.2 \%$ & 0 & $0.0 \%$ & 0 & $0.0 \%$ & 0 & $0.0 \%$ & 2 & $3.2 \%$ \\
\hline Mean sTfR (mg/L) & 4.79 & 0.22 & 4.69 & 0.23 & 4.95 & 0.22 & 5.22 & 0.26 & 4.77 & 0.25 & 4.78 & 0.26 & 4.95 & 0.26 & 4.81 & 0.26 \\
\hline $\begin{array}{l}\text { Mean iron stores } \\
(\mathrm{mg} / \mathrm{kg})\end{array}$ & 11.31 & 0.24 & 11.25 & 0.25 & 8.78 & 0.24 & 8.62 & 0.29 & 11.07 & 0.28 & 10.68 & 0.28 & 9.28 & 0.28 & 9.53 & 0.29 \\
\hline Mean RBP (umol/L) & 2.35 & 0.06 & 2.13 & 0.06 & 1.65 & 0.06 & 1.59 & 0.07 & 2.24 & 0.07 & 1.93 & 0.07 & 1.79 & 0.07 & 1.50 & 0.07 \\
\hline Vitamin A deficient $* *$ & 0 & $0.0 \%$ & 0 & $0.0 \%$ & 1 & & 0 & $0.0 \%$ & 0 & & & $1.5 \%$ & & & & $0.0 \%$ \\
\hline Mean CRP $(\mathrm{mg} / \mathrm{L})$ & 3.60 & 0.51 & 1.62 & 0.55 & 1.92 & & 1.52 & 62 & 3.71 & 0.59 & 1.79 & & 280 & & 264 & \\
\hline Elevated $(>5)$ & 14 & $15.6 \%$ & 4 & $5.1 \%$ & 8 & $8.7 \%$ & 6 & $7.1 \%$ & 12 & $17.4 \%$ & 6 & $9.2 \%$ & 8 & $11.8 \%$ & 7 & $11.3 \%$ \\
\hline Mean AGP (g & 0.67 & 0.03 & 0.61 & 0.03 & 0.57 & 0.03 & 0.56 & 0.03 & 0.72 & 0.03 & 0.65 & 0.03 & 0.69 & 0.03 & 0.59 & 0.03 \\
\hline Elevated $(>1)$ & 9 & $10.0 \%$ & 3 & $3.8 \%$ & 3 & $3.3 \%$ & 1 & $1.2 \%$ & 10 & $14.5 \%$ & 6 & $9.2 \%$ & 10 & $14.7 \%$ & 1 & $1.6 \%$ \\
\hline & Ulaan & atar & $\mathrm{Tu}$ & & $\mathrm{Bul}_{\varepsilon}$ & & Khuv & & Kho & & Omn & gobi & Sukht & aatar & Dor & \\
\hline & 77 Meas & ements & 80 Measu & ements & 81 Measu & ements & 78 Measu & ements & 77 Measu & rements & 86 Measu & ements & 79 Measu & ements & 77 Meast & rements \\
\hline & Mean or $n$ & SE or $\%$ & Mean or $n$ & SE or $\%$ & Mean or $n$ & SE or $\%$ & Mean or $n$ & SE or $\%$ & Mean or $n$ & SE or $\%$ & Mean or $n$ & SE or $\%$ & Mean or $n$ & SE or $\%$ & Mean or $n$ & SE or $\%$ \\
\hline Mean BMI (kg/m^3) & 24.6 & 0.6 & 25.7 & 0.6 & 27.8 & 0.6 & 25.8 & 0.6 & 25.2 & 0.6 & 25.0 & 0.6 & 26.3 & 0.6 & 25.6 & 0.6 \\
\hline Underweight (<18.5) & 1 & 2.5 & 0 & $0.0 \%$ & 0 & $0.0 \%$ & 0 & & 0 & $0.0 \%$ & 2 & $4.3 \%$ & 1 & & 1 & $2.5 \%$ \\
\hline Normal (18.5-25) & 22 & $55.0 \%$ & 19 & $47.5 \%$ & 9 & $19.1 \%$ & 15 & $37.5 \%$ & 18 & $43.9 \%$ & 26 & & 14 & $35.0 \%$ & 18 & $45.0 \%$ \\
\hline Overweight (25-30) & 16 & $40.0 \%$ & 16 & $\%$ & 26 & & 19 & $47.5 \%$ & 21 & $51.2 \%$ & 13 & 28. & 20 & $50.0 \%$ & 18 & $45.0 \%$ \\
\hline Obese $(30+)$ & 1 & $2.5 \%$ & 5 & $12.5 \%$ & 12 & $25.5 \%$ & 6 & $15.0 \%$ & 2 & $4.9 \%$ & 5 & $10.9 \%$ & 5 & $12.5 \%$ & 3 & $7.5 \%$ \\
\hline Mean ferritin (ug/L) & 125.7 & 8. & 130.4 & 8 & 127.2 & 7 & 107.8 & & 108.1 & 8. & 139.2 & 7.8 & 137.5 & 0 & 133.1 & 8.0 \\
\hline Iron def & 1 & $2.5 \%$ & 0 & $0.0^{\circ}$ & 1 & $2.1 \%$ & 0 & $0.0 \%$ & 2 & $4.9 \%$ & 3 & $6.5 \%$ & 0 & $0.0 \%$ & 0 & $0.0 \%$ \\
\hline Iron over & 1 & 2.5 & 0 & $0.0^{\circ}$ & 0 & $0.0 \%$ & 0 & $0.0 \%$ & 0 & $0.0 \%$ & 1 & $2.2 \%$ & 0 & $0.0 \%$ & 0 & $0.0 \%$ \\
\hline Mean sTfR (mg/L) & 4.96 & 0.32 & 4.98 & 0.32 & 4.89 & 0.30 & 5.37 & 0.32 & 4.69 & 0.32 & 4.92 & 0.31 & 4.72 & 0.32 & 4.49 & 0.32 \\
\hline $\begin{array}{l}\text { Mean iron stores } \\
(\mathrm{mg} / \mathrm{kg}) \text { st }\end{array}$ & 9.89 & 0.39 & 10.05 & 0.39 & 10.02 & 0.36 & 9.21 & 0.39 & 9.40 & 0.39 & 10.69 & 0.38 & 10.45 & 0.38 & 10.67 & 0.39 \\
\hline Mean RBP (umol/L) & 1.82 & 0.09 & 2.04 & 0.08 & 2.08 & 0.08 & 1.83 & 0.0 & 1.86 & 0.08 & 1.86 & 0.08 & 1.98 & 0.08 & 1.77 & 0.08 \\
\hline Vitamin A de & 0 & $0.0^{\circ}$ & 0 & 0.0 & 1 & $2.1 \%$ & 0 & 0. & $\begin{array}{c}1.00 \\
1\end{array}$ & 0.00 & 3 & & 0 & $0.0 \%$ & 0 & $0.0 \%$ \\
\hline Mean CRP $(\mathrm{mg} / \mathrm{L})$ & 1.63 & 0.60 & 2.62 & 0.58 & 2.49 & 0.57 & 2.49 & 0.5 & 2.29 & 0.59 & 2.55 & 0.59 & 2.33 & 0.57 & 3.02 & 0.58 \\
\hline Elevated & 1 & $2.5 \%$ & 6 & $15.0 \%$ & 4 & $8.5 \%$ & 5 & $12.5 \%$ & 4 & $9.8 \%$ & 4 & $8.7 \%$ & 3 & $7.5 \%$ & 5 & $12.5 \%$ \\
\hline Mean AGP $(\mathrm{g} / \mathrm{L})$ & 0.53 & 0.03 & 0.68 & 0.03 & 0.63 & 0.03 & 0.66 & 0.03 & 0.65 & 0.0 & 0.59 & 0.03 & 0.72 & 0.03 & 0.59 & 0.03 \\
\hline Elevated $(>1)$ & 1 & $2.5 \%$ & 5 & $12.5 \%$ & 3 & $6.4 \%$ & 4 & $10.0 \%$ & 2 & $4.9 \%$ & 0 & $0.0 \%$ & 5 & $12.5 \%$ & 0 & $0.0 \%$ \\
\hline
\end{tabular}

Within provinces, means are calculated by first averaging each person's summer and winter measurements then averaging these within-person annual means across persons in each province; provincial frequencies are based on within-person annual means. Significant differences in means $(p<0.05)$ based on least squares analysis are indicated for the following planned comparisons: summer vs. winter within urbanicity-sex (indicated in blue), males vs. females within urbanicity (BMI) or urbanicity-season (serum biomarkers) (green), urban vs. rural within sex (BMI only) or sex-season (bold), and each province vs. the mean of all other provinces (purple) (combinations of colors and/or bolding indicate multiple significant comparisons) * Seasonal means and differences in BMI are not assessed because height and weight were collected only in winter for the majority of participants. ** Iron and vitamin A deficiency are defined according to serum ferritin and RBP concentration cutoffs, respectively, which are differentially adjusted for inflammatory phase (normal, incubation, early convalescence, late convalescence) defined according to different combinations of elevated CRP and AGP ( $>5 \mathrm{mg} / \mathrm{L}$ and $>1 \mathrm{~g} / \mathrm{L}$, respectively) [21]. Iron overload is defined as serum ferritin $>300 \mathrm{ng} / \mathrm{mL}$ in men and $>200 \mathrm{ng} / \mathrm{mL}$ in women without the presence of inflammation [22]. BMI: body mass index; sTfR: soluble transferrin receptor; RBP: retinol binding protein; CRP: C-reactive protein; AGP: alpha 1-acid glycoprotein. 
Table 5. Estimated marginal means of body mass index and serum biomarker concentrations by urbanicity and age.

\begin{tabular}{|c|c|c|c|c|c|c|c|c|c|c|c|c|c|c|c|}
\hline \multirow{2}{*}{ Stratum } & \multirow{2}{*}{$\begin{array}{c}\text { Age } \\
\text { Group }\end{array}$} & \multicolumn{2}{|c|}{ BMI $\left(\mathrm{kg} / \mathrm{m}^{3}\right)$} & \multicolumn{2}{|c|}{ Ferritin (ug/L) } & \multicolumn{2}{|c|}{ sTfR (mg/L) } & \multicolumn{2}{|c|}{ Iron stores $(\mathrm{mg} / \mathrm{kg})$} & \multicolumn{2}{|c|}{ RBP (umol/L) } & \multicolumn{2}{|c|}{ CRP (mg/L) } & \multicolumn{2}{|c|}{$\operatorname{AGP}(g / L)$} \\
\hline & & Mean & SE & Mean & SE & Mean & SE & Mean & SE & Mean & SE & Mean & SE & Mean & $\mathrm{SE}$ \\
\hline Urban & \multirow{2}{*}{ All ages } & 26.4 & 0.6 & 129.2 & 6.6 & 4.88 & 0.33 & 10.15 & 0.35 & 1.95 & 0.08 & 2.23 & 0.67 & 0.61 & 0.04 \\
\hline Rural & & 24.9 & 0.6 & 122.2 & 6.8 & 4.88 & 0.35 & 9.94 & 0.36 & 1.84 & 0.08 & 2.63 & 0.68 & 0.66 & 0.04 \\
\hline \multirow{3}{*}{ Urban Male } & $<33$ & 25.8 & 0.5 & 150.5 & 5.7 & 4.88 & 0.29 & 10.93 & 0.30 & 2.12 & 0.07 & 2.50 & 0.56 & 0.64 & 0.03 \\
\hline & $33-44$ & 26.8 & 0.7 & 159.5 & 7.8 & 5.01 & 0.40 & 11.15 & 0.41 & 2.22 & 0.09 & 3.76 & 0.77 & 0.62 & 0.04 \\
\hline & $45+$ & 28.2 & 0.8 & 177.6 & 7.9 & 4.22 & 0.40 & 12.04 & 0.42 & 2.47 & 0.09 & 1.83 & 0.76 & 0.66 & 0.04 \\
\hline \multirow{3}{*}{ Urban Female } & $<33$ & 24.4 & 0.6 & 74.8 & 6.9 & 5.61 & 0.35 & 7.49 & 0.37 & 1.61 & 0.08 & 1.86 & 0.69 & 0.59 & 0.04 \\
\hline & $33-44$ & 25.0 & 0.5 & 73.7 & 6.4 & 5.11 & 0.33 & 8.03 & 0.34 & 1.64 & 0.08 & 1.51 & 0.65 & 0.57 & 0.04 \\
\hline & $45+$ & 27.1 & 0.6 & 129.3 & 6.6 & 4.76 & 0.33 & 10.45 & 0.35 & 1.65 & 0.08 & 1.75 & 0.64 & 0.53 & 0.04 \\
\hline \multirow{3}{*}{ Rural Male } & $<33$ & 25.9 & 0.7 & 131.2 & 9.6 & 4.55 & 0.49 & 10.59 & 0.51 & 2.07 & 0.11 & 2.04 & 0.94 & 0.82 & 0.05 \\
\hline & $33-44$ & 26.0 & 0.6 & 151.6 & 7.2 & 5.30 & 0.36 & 10.75 & 0.38 & 2.07 & 0.08 & 2.98 & 0.71 & 0.66 & 0.04 \\
\hline & $45+$ & 26.1 & 0.6 & 147.4 & 7.1 & 4.29 & 0.36 & 11.15 & 0.37 & 2.09 & 0.08 & 2.95 & 0.70 & 0.64 & 0.04 \\
\hline \multirow{3}{*}{ Rural Female } & $<33$ & 24.4 & 0.7 & 79.0 & 9.2 & 4.98 & 0.47 & 7.84 & 0.48 & 1.70 & 0.11 & 2.54 & 0.91 & 0.60 & 0.05 \\
\hline & $33-44$ & 24.6 & 0.6 & 103.2 & 6.8 & 5.02 & 0.35 & 9.46 & 0.36 & 1.57 & 0.08 & 2.44 & 0.68 & 0.70 & 0.04 \\
\hline & $45+$ & 24.8 & 0.6 & 128.6 & 7.8 & 4.60 & 0.40 & 10.41 & 0.41 & 1.69 & 0.09 & 3.12 & 0.76 & 0.61 & 0.04 \\
\hline
\end{tabular}

In urban vs. rural analysis, mean BMI, CRP, and AGP are adjusted for age in years, sex, province, season, and a random intercept per person to account for within-person correlation across seasons. In analysis of age, mean BMI, CRP, and AGP are estimated with a model in which the outcome in either season is predicted by an age group $\times$ urbanicity-sex group interaction term adjusted for province, season, and a random intercept per person. Within urbanicity-sex groups, underlined type indicates a significant linear trend with age ( $p<0.05)$. Significant differences in trends are indicated for the following planned comparisons: urban vs. rural (or, in analysis of age, urban vs. rural within sex) (indicated in bold) and males vs. females within urbanicity (green). In both urbanicity and age analyses, mean ferritin, sTfR, body iron stores, and RBP are additionally adjusted for CRP and AGP. BMI: body mass index; sTfR: soluble transferrin receptor; RBP: retinol binding protein; CRP: C-reactive protein; AGP: alpha 1-acid glycoprotein. 
Table 6. Diet pattern factor loadings and mean intake (g/day) of pattern components by quintile of pattern scores.

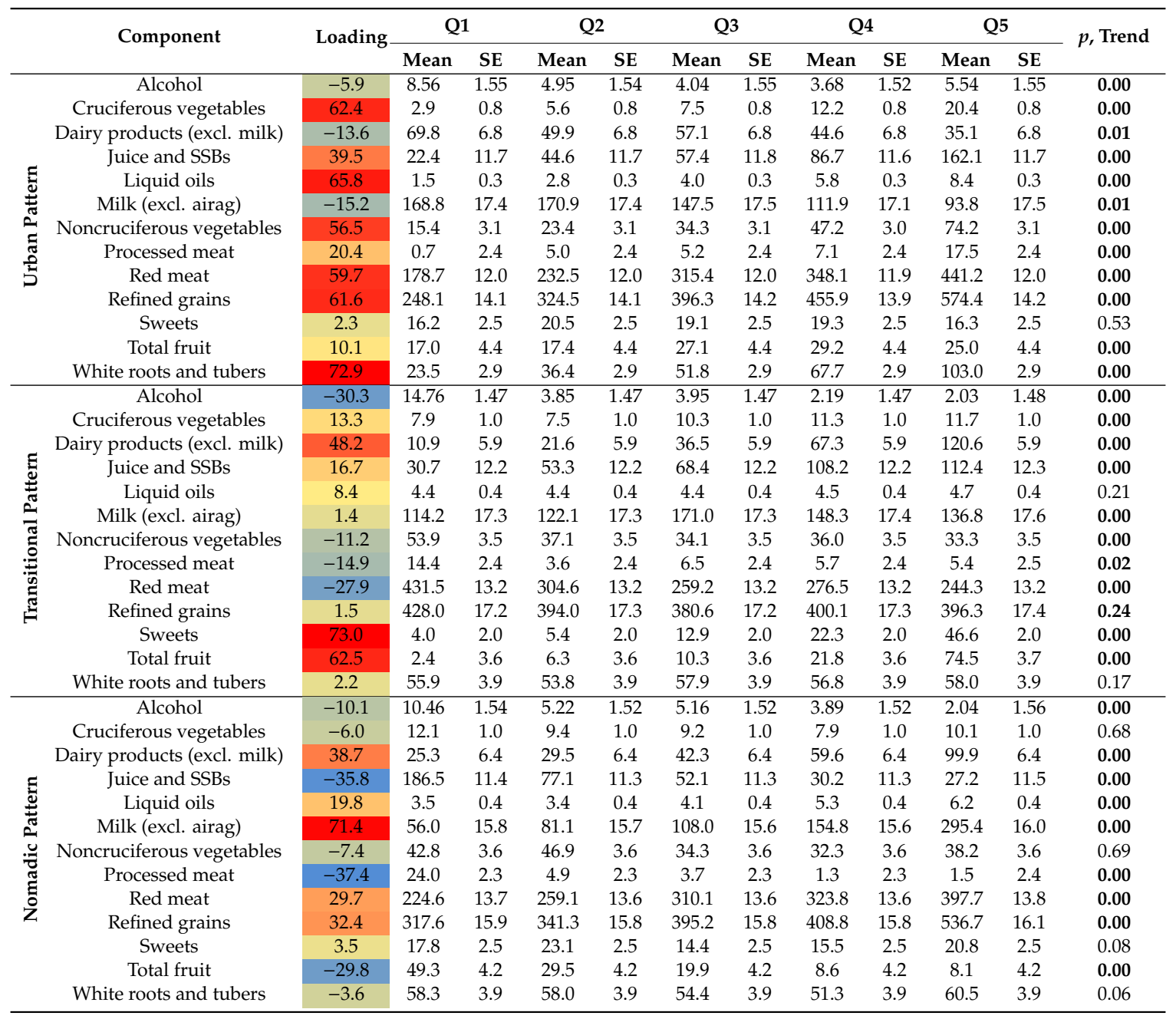

Urban, Transitional, and Nomadic diet patterns account for $20.5 \%, 10.9 \%$, and $9.8 \%$ of variation in intake of pattern components, respectively (total: $41.1 \%$ ). Factor loadings are generated using annualized average intakes for 334 participants (based on 1834 diet record days); used to calculate season-specific patterns scores for each participant; and seasonal pattern scores are scaled from 0-100. Mean intakes of pattern components within quintiles are also calculated based on annualized averages, and are adjusted for a random intercept per person to account for within-person correlation across seasons. Shading is proportional to magnitude and direction of factor loading (blue: $-37.4 \%$, yellow: $0 \%$, red: $73.0 \%$ ). Bold type indicates $p<0.5$. 


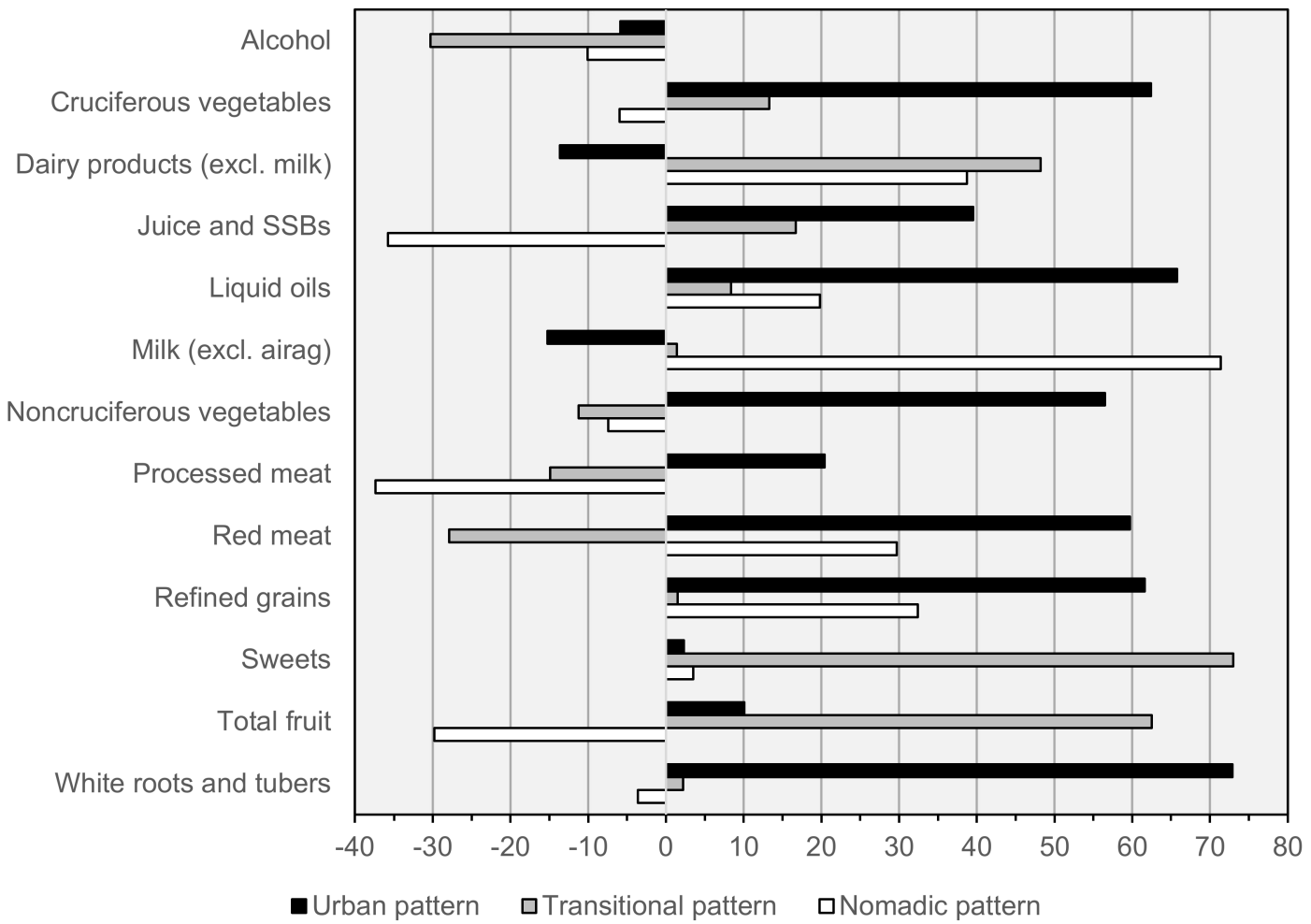

Figure 2. Diet pattern factor loadings. Urban, Transitional, and Nomadic diet patterns account for $20.5 \%, 10.9 \%$, and $9.8 \%$ of variation in intake of pattern components, respectively (total: $41.1 \%$ ). Factor loadings are generated using annualized average intakes for 334 participants (based on 1834 diet record days); used to calculate season-specific patterns scores for each participant; and seasonal pattern scores are scaled from 0-100. Airag: fermented mares' milk, SSBs: sugar-sweetened beverages.

Table 7. Mean diet pattern scores by subgroup-season and province.

\begin{tabular}{|c|c|c|c|c|c|c|}
\hline \multirow{2}{*}{ Stratum } & \multicolumn{2}{|c|}{ Urban Pattern Score } & \multicolumn{2}{|c|}{ Transitional Pattern Score } & \multicolumn{2}{|c|}{ Nomadic Pattern Score } \\
\hline & Mean & SE & Mean & SE & Mean & SE \\
\hline Urban Male Summer & 38.3 & 1.6 & 27.2 & 0.9 & 20.6 & 0.7 \\
\hline Urban Male Winter & 36.1 & 1.7 & 24.0 & 1.0 & 21.5 & 0.7 \\
\hline Urban Female Summer & 27.0 & 1.6 & 33.5 & 0.9 & 21.5 & 0.7 \\
\hline Urban Female Winter & 23.6 & 1.7 & 32.0 & 1.0 & 20.5 & 0.7 \\
\hline Rural Male Summer & 25.0 & 1.8 & 24.7 & 1.0 & 27.6 & 0.8 \\
\hline Rural Male Winter & 30.9 & 1.8 & 22.2 & 1.0 & 29.1 & 0.8 \\
\hline Rural Female Summer & 16.1 & 1.8 & 28.9 & 1.0 & 25.3 & 0.8 \\
\hline Rural Female Winter & 19.6 & 1.9 & 26.0 & 1.1 & 24.6 & 0.8 \\
\hline Ulaanbaatar & 37.2 & 2.1 & 28.7 & 1.2 & 20.7 & 0.9 \\
\hline Tuv & 28.7 & 2.1 & 24.6 & 1.2 & 23.0 & 0.9 \\
\hline Bulgan & 24.2 & 2.0 & 27.7 & 1.1 & 21.9 & 0.9 \\
\hline Khovd & 26.2 & 2.1 & 28.8 & 1.2 & 25.5 & 0.9 \\
\hline Khuvsgul & 26.8 & 2.0 & 27.1 & 1.2 & 24.9 & 0.9 \\
\hline Omnogobi & 26.5 & 2.0 & 29.4 & 1.1 & 22.3 & 0.9 \\
\hline Sukhbaatar & 24.5 & 2.0 & 26.6 & 1.2 & 25.2 & 0.9 \\
\hline Dornod & 26.7 & 2.1 & 28.1 & 1.2 & 24.7 & 0.9 \\
\hline
\end{tabular}

Within provinces, means are calculated by first averaging each person's summer and winter pattern scores and then averaging these within-person annual means across persons in each province. Significant differences in means $(p$ $<0.05$ ) based on least squares analysis are indicated for the following planned comparisons: summer vs. winter within urbanicity-sex (indicated in blue), males vs. females within urbanicity-season (green), urban vs. rural within sex-season (bold), and each province vs. the mean of all other provinces (purple) (combinations of colors and/or bolding indicate multiple significant comparisons). 
Table 8. Estimated marginal means of diet pattern scores by age.

\begin{tabular}{lccccccc}
\hline \multirow{2}{*}{ Urbanicity-Sex } & \multirow{2}{*}{ Age Group } & \multicolumn{2}{c}{ Urban Pattern Score } & \multicolumn{2}{c}{ Transitional Pattern Score } & \multicolumn{2}{c}{ Nomadic Pattern Score } \\
\cline { 2 - 7 } & & Mean & SE & Mean & SE & Mean & SE \\
\hline \multirow{3}{*}{ Urban Male } & $<33$ & 37.0 & 1.4 & 25.6 & 1.0 & 19.4 & 0.7 \\
& $33-44$ & 35.2 & 2.0 & 24.5 & 1.4 & 20.4 & 1.0 \\
Urban & $45+$ & 31.2 & 2.0 & 25.3 & 1.4 & 22.3 & 1.0 \\
Female & $<33$ & 31.0 & 1.8 & $\mathbf{3 2 . 7}$ & 1.3 & 21.1 & 0.9 \\
& $33-44$ & 29.6 & 1.7 & $\mathbf{3 2 . 2}$ & 1.1 & 23.0 & 0.8 \\
Rural Male & $45+$ & 25.6 & 1.7 & 34.7 & 1.1 & 21.7 & 0.8 \\
& $<33$ & 25.4 & 2.3 & 22.0 & 1.6 & 24.9 & 1.1 \\
\multirow{2}{*}{ Rural Female } & $33-44$ & 25.3 & 1.8 & 23.0 & 1.2 & 26.8 & 0.9 \\
& $45+$ & 19.6 & 1.8 & 22.8 & 1.2 & 28.4 & 0.9 \\
& $33-44$ & 20.3 & 2.3 & $\mathbf{3 0 . 8}$ & 1.6 & 24.6 & 1.2 \\
& $45+$ & 20.4 & 1.7 & $\mathbf{2 7 . 5}$ & 1.2 & 26.0 & 0.8 \\
\end{tabular}

Means are estimated using a regression model in which summer or winter pattern score $(n=635)$ is predicted using an age group $\times$ urbanicity-sex group interaction term adjusted for province, season, within-season mean daily energy intake, and a random intercept per person to account for within-person correlation across seasons. Within urbanicity-sex groups, underlined type indicates a significant linear trend with age $(p<0.05)$. Significant differences in trends are indicated for the following planned comparisons: urban vs. rural within sex (indicated in bold) and males vs. females within urbanicity (green).

Trends in estimated marginal means of energy intake and body mass index by age and pattern scores across subgroups are presented graphically in Figure 4. Despite no significant differences in linear trend of adjusted energy intake with age between any population subgroups, a significant upward trend in BMI with age was observed among both men and women in urban areas but not rural areas (Figure 4, Table 5). Increased adherence to all pattern scores was independently associated with increased energy intake, however only the Urban pattern was also (positively) associated with increased BMI (Figure 4, Table 9). 

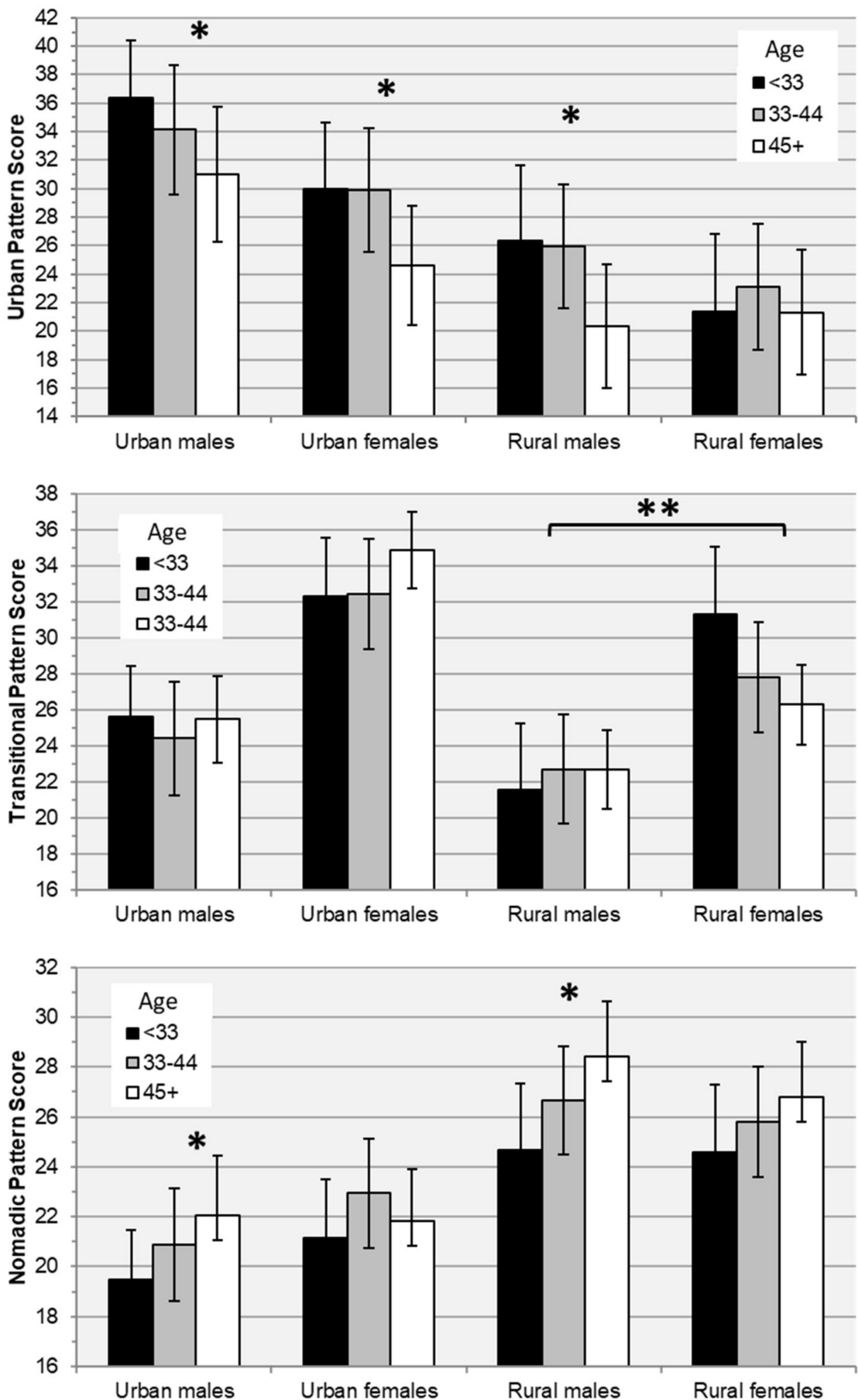

Figure 3. Age-trends in estimated marginal means of diet pattern scores by subgroup. Means are estimated using a regression model in which summer or winter pattern score $(n=635)$ is predicted using an age group $\times$ urbanicity-sex group interaction term adjusted for province, season, within-season mean daily energy intake, and a random intercept per person to account for within-person correlation across seasons. Error bars indicate 95\% confidence intervals. * Significant linear trend with age $(p<$ 0.05). ${ }^{* *}$ Significant difference in age trends between rural males and females. 
Table 9. Estimated marginal means of selected nutrient intakes and clinical measurements by quintile of diet pattern scores.

\begin{tabular}{|c|c|c|c|c|c|c|c|c|c|c|c|c|c|c|c|c|c|c|c|c|c|c|c|}
\hline \multirow{3}{*}{\multicolumn{2}{|c|}{ Measurement }} & \multicolumn{10}{|c|}{ Energy-Unadjusted } & \multirow{3}{*}{$p$, Trend } & \multicolumn{10}{|c|}{ Energy-Adjusted } & \multirow{3}{*}{$p$, Trend } \\
\hline & & \multicolumn{2}{|c|}{ Q1 } & \multicolumn{2}{|c|}{ Q2 } & \multicolumn{2}{|c|}{ Q3 } & \multicolumn{2}{|c|}{ Q4 } & \multicolumn{2}{|c|}{ Q5 } & & \multicolumn{2}{|c|}{ Q1 } & \multicolumn{2}{|c|}{ Q2 } & \multicolumn{2}{|c|}{ Q3 } & \multicolumn{2}{|c|}{ Q4 } & \multicolumn{2}{|c|}{ Q5 } & \\
\hline & & Mean & SE & Mean & SE & Mean & SE & Mean & SE & Mean & SE & & Mean & SE & Mean & SE & Mean & SE & Mean & SE & Mean & SE & \\
\hline & Energy (kcal) & 1809 & 117 & 2181 & 118 & 2561 & 119 & 2726 & 125 & 3391 & 134 & 0.00 & & & & & & & & & & & \\
\hline & Protein $(g)$ & 93.5 & 6.8 & 110.1 & 6.8 & 133.0 & 6.9 & 141.5 & 7.3 & 178.4 & 7.8 & 0.00 & 126.1 & 4.5 & 125.0 & 4.4 & 130.9 & 4.4 & 31.4 & 4.7 & 137.9 & 5.1 & 0.01 \\
\hline & Fiber (g) & 5.6 & 0.6 & 7.5 & 0.6 & 9.6 & 0.6 & 11.1 & 0.6 & 14.1 & 0.6 & 0.00 & 7.7 & 0.5 & 8.4 & 0.5 & 9.4 & $\begin{array}{l}.4 \\
0.5\end{array}$ & 0.5 & 0.5 & 11.6 & 0.5 & 0.00 \\
\hline & Calcium (mg) & 859 & 108 & 718 & 109 & 674 & 110 & 636 & 117 & 695 & 125 & 0.06 & 1103 & 106 & 829 & 103 & 660 & 102 & 561 & 109 & 391 & 119 & 0.00 \\
\hline & Iron (mg) & 11.48 & 1.04 & 14.56 & 1.04 & 18.10 & 1.06 & 19.34 & 1.12 & 25.52 & 1.20 & 0.00 & 14.85 & 0.90 & 16.10 & 0.87 & 17.89 & 0.87 & 18.28 & 0.93 & 21.34 & 1.02 & 0.00 \\
\hline & Zinc (mg) & 13.21 & 1.02 & 15.97 & 1.02 & 20.04 & 1.03 & 21.12 & 1.10 & 26.92 & 1.17 & 0.00 & 17.17 & 0.83 & 17.78 & 0.81 & 19.80 & 0.81 & 19.91 & 0.85 & 21.99 & 0.94 & 0.00 \\
\hline & itamin A (ug retinol eq.)) & 506 & 210 & 753 & 211 & 568 & 213 & 620 & 227 & 1130 & 243 & 01 & 652 & 219 & 817 & 213 & 560 & 212 & 576 & 226 & 951 & 248 & 0.61 \\
\hline & Folate (ug DFE) & 107 & 14 & 134 & 14 & 148 & 14 & 155 & 15 & 205 & 16 & 00 & 144 & 13 & 152 & 13 & 145 & 13 & 143 & 14 & 159 & 15 & 0.56 \\
\hline & Vitamin B12 (ug) & 9.86 & 3.26 & 11.97 & 3.28 & 13.85 & 3.31 & 13.18 & 3.53 & 21.40 & 3.77 & 0.00 & 12.26 & 3.40 & 13.03 & 3.30 & 13.72 & 3.29 & 12.44 & 3.51 & 18.44 & 3.85 & 0.19 \\
\hline & BMI $\left(\mathrm{kg} / \mathrm{m}^{3}\right)$ & 25.4 & 0.6 & 25.5 & 0.6 & 25.6 & 0.6 & 25.8 & 0.6 & 25.9 & 0.6 & .00 & 25.3 & 0.6 & 25.5 & 0.6 & 25.6 & 0.6 & 25.8 & 0.6 & 26.0 & 0.6 & 0.00 \\
\hline & & 129.8 & 7.3 & 127.2 & 7.3 & 123.8 & 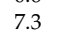 & 124.6 & 7. & & 7.8 & & & 7.4 & 20.0 & 7.4 & & & 5.1 & & & & 0.46 \\
\hline & sT & 4.79 & 0.38 & 4.85 & 0.38 & 5.06 & 0.38 & 5.02 & 0.39 & 36 & 0.40 & & 88 & 0.39 & 4.90 & 0.38 & 05 & 0.38 & 00 & 39 & 75 & & 88 \\
\hline & Iron stores (r & 10.25 & 0.38 & 10.19 & 0.39 & 9.85 & 0.39 & 9.96 & 0.39 & 9.92 & 0.41 & 88 & .17 & 0.39 & 10.14 & 0.39 & 9.86 & 0.38 & 98 & 0.39 & 10.03 & 0.41 & 0.51 \\
\hline & $\mathrm{RBP}(\mathrm{umol} / \mathrm{L})$ & 1.87 & 0.09 & 1.84 & 0.09 & 1.90 & 0.09 & 1.88 & 0.09 & 1.94 & 0.10 & 0.28 & 1.85 & 0.09 & 1.83 & 0.09 & 1.90 & 0.09 & 1.89 & 0.09 & 1.97 & 0.10 & 0.19 \\
\hline & Energy (kcal) & 2865 & 137 & 2495 & 136 & 2373 & 136 & 2329 & 136 & 2587 & 147 & 0.01 & & & & & & & & & & & \\
\hline & $\mathrm{P}_{1}$ & 134.9 & 7.9 & 122.9 & 7. & 122.2 & 7.9 & 122.6 & 7.8 & 6 & & & 7.5 & 2 & 23.7 & 4.2 & 9.2 & 4.2 & 1.5 & 2 & 47.7 & .5 & 00 \\
\hline & $\mathrm{Fi}$ & 11 & 0.7 & 9.1 & 0.7 & 8 & 0 & 9.0 & 0 & & & & & & & & & & & & & & 1 \\
\hline & Calcium (mg) & 849 & 110 & 718 & 109 & 703 & 109 & 565 & 109 & 763 & 118 & 0.1 & 777 & 107 & 721 & 107 & 731 & 108 & 602 & 08 & 748 & 115 & 41 \\
\hline & Iron (mg) & 17.95 & 1.27 & 17.59 & 1.26 & 16.04 & 1.26 & 16.95 & 1.26 & 20.11 & 1.36 & 0.14 & 15.76 & 0.90 & 17.71 & 0.90 & 16.85 & 0.91 & 18.09 & 0.91 & 19.66 & 0.97 & 0.00 \\
\hline & Zinc (mg) & 19.07 & 1.20 & 18.21 & 1.19 & 17.72 & 1.19 & 18.36 & 1.19 & 23.46 & 1.29 & 0.00 & 16.70 & 0.78 & 18.33 & 0.78 & 18.67 & 0.79 & 19.56 & 0.79 & 22.95 & 0.84 & 0.00 \\
\hline & Vitamin A (ug retinol eq.)) & 767 & 218 & 801 & 216 & 617 & 217 & 766 & 216 & 60.40 & 234 & 0.00 & 10.70 & & 806 & 215 & 10.07 & (1) & 1.00 & . & 602 & 233 & 0.73 \\
\hline & & 15 & 15 & 1 & 1 & 13 & 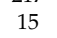 & 14 & 1 & & 20 & & & 13 & & & & & & & & & \\
\hline & Vitamin B12 (ug) & 13.73 & 3.38 & 13.89 & 3.35 & 11.25 & 3.37 & 13.86 & 3.36 & 17.22 & 3.64 & 0.3 & 12.07 & 3.33 & 13.99 & 3.33 & 11.89 & 3.38 & 14.71 & 3.38 & 16.89 & 3.61 & 0.12 \\
\hline & & 25.9 & 0.6 & 25.5 & 0.6 & 25.5 & 0.6 & 25.6 & 0.6 & 25.7 & 0.6 & 0. & 25.9 & 0.6 & 25.5 & 0.6 & 25.5 & 0.6 & 25.6 & 0.6 & 25.7 & 0.6 & 0.54 \\
\hline & & 125.7 & 7.4 & 126.8 & 7.3 & 126.0 & 7.3 & 127.2 & 7.4 & 121.9 & 7.6 & & 126.5 & 7.4 & 126.9 & 7.3 & 125.6 & 7.4 & 126.8 & 4 & 122.2 & 7.6 & 33 \\
\hline & & 4.98 & 0.38 & 5.08 & 0.38 & 4.63 & 0.38 & 4.90 & 0.39 & 5.02 & 0.39 & 0 & & 0.38 & 5.07 & 0.38 & 4.64 & 0.39 & 91 & 0.39 & 5.01 & 39 & 98 \\
\hline & Iron st & 10.08 & 0.39 & 10.06 & 0.3 & 10.14 & 0.39 & 10.06 & 0.3 & & & & & & 10.07 & & 10.12 & & 10.04 & & & & 18 \\
\hline & RBP (umol/ $/$ & 1.86 & 0.09 & 1.83 & 0.09 & 1.99 & 0.09 & 1.86 & 0.09 & 1.90 & 0.10 & 0.46 & 1.86 & 0.09 & 1.83 & 0.09 & 1.99 & 0.09 & 1.86 & 0.09 & 1.90 & 0.10 & 0.45 \\
\hline & Energy (kcal) & 2043 & 127 & 2178 & 121 & 2359 & 121 & 2610 & 119 & 3343 & 128 & 0.0 & & & & & & & & & & & \\
\hline & & 104.5 & 7.6 & 114.7 & 7 & 123.0 & 7.3 & 135.7 & 7. & & & & 126.6 & 4.8 & 130.6 & 4.6 & 130.6 & 4.6 & 1.0 & 4.4 & 131.7 & 4.7 & 0.25 \\
\hline & & 8.8 & 0.7 & 8.9 & 0. & 8. & 0. & 9.4 & 0. & & & & & 0.5 & 10 & 0.5 & & & 9.0 & & 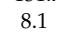 & 0.5 & 00 \\
\hline & Calcium (mg) & 714 & 114 & 68 & 10 & 64 & 10 & 594 & 10 & & 115 & 0. & & 115 & & 109 & & 8 & 573 & 4 & 756 & 12 & 0.12 \\
\hline & & 13.79 & 1.21 & 15. & 1. & 16. & 1. & 18.62 & 1. & & 1. & & & & 17 & 0.94 & 17.27 & 0.93 & 8.04 & 0.90 & 19.16 & & .00 \\
\hline & $\mathrm{Zir}$ & 14.93 & 1.1 & 17. & 1.1 & 18 & 1 . & 20 & 1. & & 1. & & 17.78 & 0.91 & 19 & 0.86 & 19.47 & 0.85 & 19.62 & 0.83 & 20.21 & 39 & 00 \\
\hline & $\operatorname{tamin} \mathrm{A}(\mathrm{u}$ & 51 & 225 & 61 & 21 & 58 & 2 & 71 & 21 & & 227 & & & & 680 & 22 & & 219 & 694 & 211 & 933 & 228 & 0.23 \\
\hline & Folate (ug DF & 123 & 15 & 128 & 14 & 139 & 14 & 148 & 14 & 20 & 15 & 0.0 & 14 & 14 & 145 & 13 & 14 & 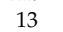 & 144 & 13 & 160 & 14 & 0.50 \\
\hline & Vitamin B12 (ug & 10.56 & $\begin{array}{l}139 \\
3.49\end{array}$ & 11.58 & $\begin{array}{l}14 \\
3.33\end{array}$ & 11.77 & $\begin{array}{l}14 \\
3.34\end{array}$ & $\begin{array}{l}140 \\
13.57\end{array}$ & $\begin{array}{l}14 \\
3.26\end{array}$ & 21.77 & 3.51 & 0.00 & $\begin{array}{l}147 \\
12.09\end{array}$ & $\begin{array}{l}14 \\
3.61\end{array}$ & 12.67 & $\begin{array}{l}13 \\
3.42\end{array}$ & 12.27 & $\begin{array}{l}10 \\
3.38\end{array}$ & $\begin{array}{l}144 \\
13.25\end{array}$ & 3.26 & 19.07 & $\begin{array}{l}14 \\
3.52\end{array}$ & 09 \\
\hline & & 25. & 0.6 & 25.7 & 0.6 & 25.5 & 0.6 & 25. & 0. & 25 & 0. & & & 0.6 & 25. & 0.6 & 25.5 & 0.6 & 25.6 & & 25.5 & 0.6 & 0.07 \\
\hline & & 12 & 7 & 12 & 0. & 12 & 7 & 12 & 0. & & 0 & & & $=$ & & 0. & 125.9 & & 24.3 & & 30.8 & 7.5 & 0.13 \\
\hline & & 4.77 & 0.39 & 5.0 & 0.38 & 5.02 & 0.38 & 4.97 & 0.38 & 4.7 & 0.39 & 0.9 & 4. & 0.40 & 5. & 0.39 & 5.03 & 0.38 & 4.96 & 0.38 & 4.68 & 0.39 & 0.58 \\
\hline & Iron st & 10.04 & 0.39 & 10.08 & 0.39 & 10.00 & 0.39 & 9.93 & 0.38 & 10.13 & 0.40 & 0.94 & 9.95 & 0.40 & 10.02 & 0.39 & 9.98 & 0.39 & 9.94 & 0.38 & 10.29 & 0.40 & 0.34 \\
\hline & $\mathrm{RBP}(\mathrm{umol} / \mathrm{L})$ & 1.87 & 0.09 & 1.87 & 0.09 & 1.93 & 0.09 & 1.87 & 0.09 & 1.90 & 0.10 & 0.73 & 1.87 & 0.10 & 1.87 & 0.09 & 1.93 & 0.09 & 1.87 & 0.09 & 1.90 & 0.10 & 0.69 \\
\hline
\end{tabular}

For each diet pattern, means are estimated using a regression model in which summer or winter mean nutrient intake, biomarker $(n=635)$, or BMI $(n=334)$ are predicted by quintile of pattern score adjusting for age in years, sex, urbanicity, and province; nutrient intake and biomarker models are additionally adjusted for season and a random intercept per person to account for within-person correlation across seasons. Energy-adjusted means are further adjusted for within-season mean daily energy intake. Bold type indicates $p<0.5$. BMI: body mass index; sTfR: soluble transferrin receptor; RBP: retinol binding protein. 

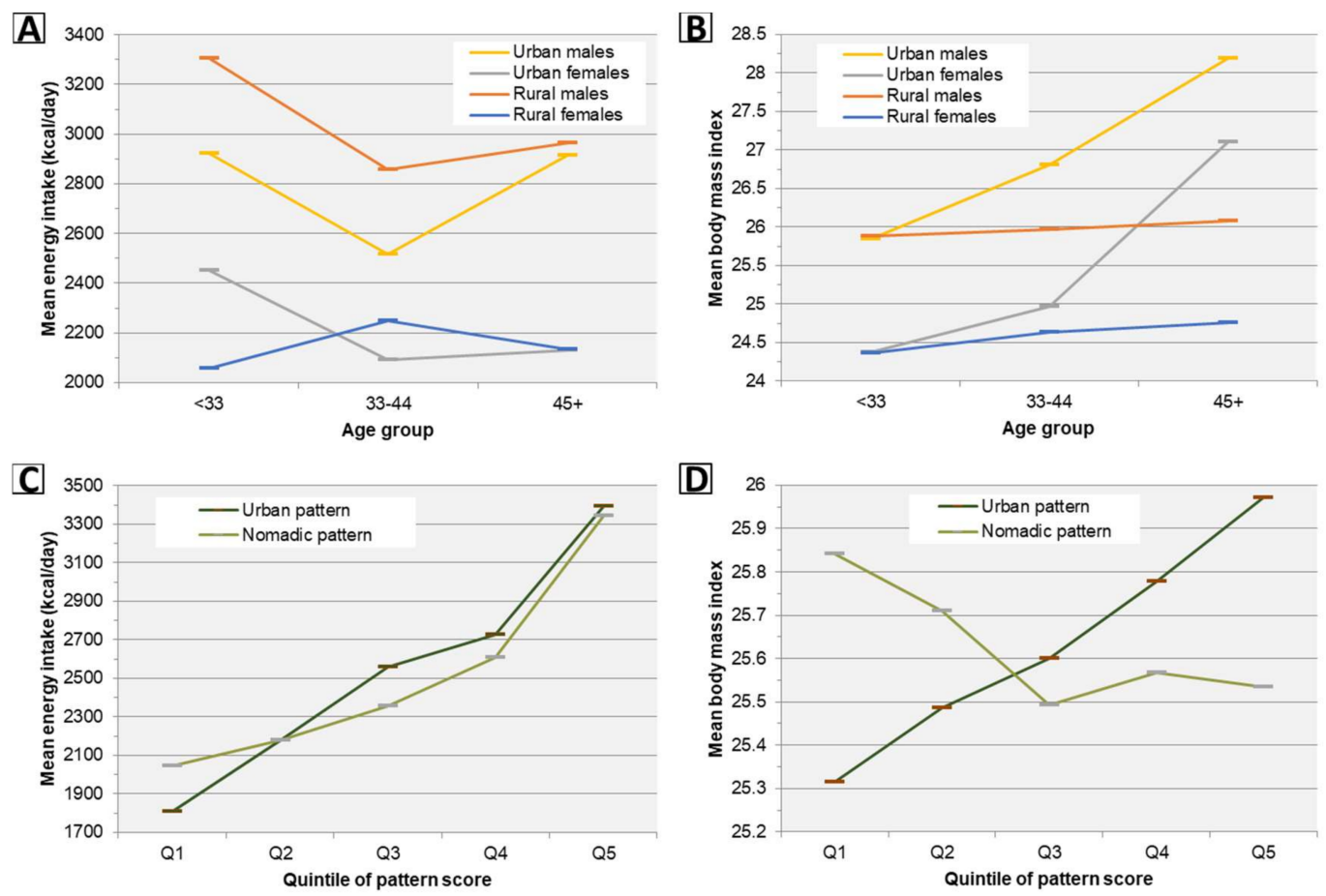

Figure 4. Trends in estimated marginal means of energy intake and body mass index by age and diet pattern scores across subgroups. (A,B) Means are estimated using a regression model in which summer or winter mean energy intake $(n=635)$ or BMI $(n=334)$ are predicted using an age group $\times$ urbanicity-sex group interaction term adjusted for province; energy models are additionally adjusted for season and a random intercept per person to account for within-person correlation across seasons. Within both urban males and rural males, adjusted energy intake decreases significantly $(p<0.05)$ from $<33$ to 33-44 years, and there are no significant differences in linear age trends between any subgroups; see Table 5 for age trends in BMI. (C,D) For each diet pattern, means are estimated using a regression model in which summer or winter mean energy intake $(n=635)$ or BMI $(n=334)$ are adjusted for quintile of pattern score, age in years, sex, urbanicity, and province; energy models are additionally adjusted season and a random intercept per person. See Table 9 for trends in energy intake and BMI across quintiles of pattern scores.

\section{Discussion}

The present study found a high prevalence of key dietary nutrient inadequacies in a nationwide sample of Mongolian adults in summer and winter. Prior analysis of data collected from the present study population, and the Fifth National Nutrition Survey (FNNS), indicate an extremely high prevalence of biochemical vitamin D deficiency throughout the Mongolian population, especially in winter [7,9]. The present study also found a high prevalence of dietary vitamin A inadequacy, and among women, a moderate prevalence of iron inadequacy. By contrast, we found little evidence of biochemical iron and vitamin A deficiency; in the FNNS, these deficiencies were also rare, while iron deficiency was moderately prevalent among pregnant women [7]. Retinol binding protein, while useful for assessing clinical vitamin A deficiency, is subject to homeostatic regulation that renders it a less sensitive measure of subclinical deficiency (which may be widespread in Mongolia, given the high prevalence of dietary vitamin A inadequacy observed in the present study). There is also evidence that biochemical deficiencies disproportionately affect young children and pregnant women in Mongolia [7,28,34].

Other nutrients of concern identified in our study included fiber, calcium, magnesium, thiamin, folate, and vitamins B6, C, D, and E, while inadequacies of protein, zinc, riboflavin, niacin, and vitamin B12 were uncommon. With the exception of calcium, these findings can generally be attributed to the population's low consumption of nutrient-dense vegetal foods (including fruits, non-tuberous 
vegetables, and whole grains) and high consumption of animal-source foods. Low calcium intake was more common in urban areas and in winter given lower urban and winter consumption of milk and dairy products. Inadequate intake of calcium, vitamin D, and magnesium may jointly contribute to the relatively high prevalence of osteoporosis in Mongolian adults [35], and low intake of fiber has been linked to higher risk of heart disease, type 2 diabetes, and metabolic syndrome in large prospective cohort studies [36-38]. Implications of observed deficits of folate and other nutrients are discussed in previous studies by our group [16], in which we have also suggested evidence-based interventions to reduce the prevalence of micronutrient deficiency in the Mongolian population, including industrial food fortification and micronutrient supplementation $[9,16,39]$. As micronutrients work together to allow healthy body functioning and prevent disease, addressing multiple inadequacies (particularly through large-scale diet modification in the long term) may reap multiplicative benefits, and ensuring adequate micronutrient intake should remain integral to Mongolia's national health policy.

It is notable that fat contributed an average of $38 \%$ of caloric energy in this survey. Although we lacked data on the breakdown of fatty acid intake, as the majority of fat consumed originated from meat and dairy products, it can be assumed that saturated fat contributed a majority of fat consumed; this is supported by results from the Global Dietary Database [40]. Omega-3 fatty acid intake was also likely very low in the study population, given minimal observed intake of fish, nuts, and seeds, and the relatively low alpha-linolenic content of the dominant liquid oil consumed (sunflower seed oil) [41]. Low intake of unsaturated fats has been associated with a more deleterious cholesterol profile, insulin resistance, and higher blood pressure [42-45]; this, and combination with a constellation of other dietary risks for chronic disease observed in our study (including high intake of refined grains, red meat, and high intake of sugary drinks in urban areas) are likely important contributors to the increasing burden of cardiovascular disease and type 2 diabetes in Mongolia [6-8].

In the present study, three dietary patterns explained $41 \%$ variation in consumption of major food groups, and while all were associated with increased energy intake, only the "Urban" pattern was independently associated with increased body mass index. In a prior study, Dugee and colleagues derived three exploratory diet patterns using semiquantitative food frequency questionnaire data collected from 420 healthy men and women aged $>25$ years from urban and rural areas of Ulaanbaatar and Khuvsgul in August 2005 [46], providing an interesting comparison with Mongolian adults in the present study in 2012-2016. Between 2005 and 2012-2016, pattern factor loadings and the variation in food intake explained by each were closely comparable, except that the 2012-2016 "Transitional" pattern contains high factor loadings for both sweets and SSBs not present in the comparable 2005 "Healthy" pattern. Likely in part due to this difference, only the 2005 "Healthy" pattern, and not its 2012-2016 "Transitional" counterpart, was associated with reduced odds of obesity in energy-adjusted analysis. Both the 2005 study's "Transitional" pattern and its 2012-2016 "Urban" counterpart were associated with obesity in both unadjusted and energy-adjusted analyses, while the 2005 "Traditional" pattern and its 2012-2016 "Nomadic" counterpart were not, suggesting that diets in urban Mongolia have remained consistently obesogenic, while that of rural areas are not. Furthermore, our finding that adherence to the 2012-2016 "Urban" and "Nomadic" patterns decreases and increases with age, respectively, in both urban and rural areas suggests the possibility that the nation will continue to converge toward an increasingly obesogenic diet in the future.

A key finding of this study was an upward trend in BMI by age among urban Mongolian adults, but not rural ones, despite similar age trends in energy intake. One probable reason for this difference is an intensely physically-active lifestyle that many nomadic Mongolians continue to engage in even into older age, while lifestyles in urban areas have become increasingly sedentary [8]. Another likely reason is higher adherence in urban areas to the Urban diet pattern, which was positively associated with BMI after adjustment for age and energy intake. It is known that increased consumption of specific foods typically associated with industrial or western diet patterns globally (such as refined grain products and sugar-sweetened beverages, factor loadings of which were highest for the Urban pattern) are linked to long-term weight gain independent of increases in energy intake [47]. 
Comparable to results of the FNNS, the present study observed a low prevalence of underweight and a high prevalence of overweight and obesity among urban and rural men and non-pregnant women [7]. Nationally, from 2005 to 2013 alone, the prevalence of child stunting decreased from 27.5 to $10.8 \%$ [1], while that of overweight increased more than all but three countries globally [48]. In addition, analysis of Multiple Indicator Cluster Survey (MICS) data from 2005, 2009, and 2013 has shown consistent increases in population mean body mass index, waist circumference, percent body fat, blood pressure, and cholesterol in the adult population [8], with $61.9 \%$ and $27.5 \%$ of Mongolian adults having elevated levels of serum cholesterol and blood pressure, respectively, or on blood pressure medication. Concurrent to trends in metabolic risks, 2005 to 2013 saw a 11.4\% increase in per capita caloric energy in the Mongolian Food Supply (the 15th largest increase among 175 countries during that period) and an increase in the prevalence of low physical activity ( $<600$ MET-minutes/week) from 7.4 to $22.3 \%[2,8]$, trends that are in turn plausibly related to tremendous economic and demographic changes that have swept Mongolia as a result of the country's recent mining boom. Since the year 2000, the fraction of Mongolians living in urban areas increased from 57.1 to $68.4 \%$, national gross domestic production by a factor of 11.4, and total imports value by a factor of 9.6 [1]. While rapid economic growth and foreign investment, globalization of the food market, and shifts from a traditionally nomadic lifestyle to one of urban sedentism are implicated in Mongolia's waning burden of severe undernutrition, these trends have also contributed toward the proliferation of noncommunicable diseases and have done so since the country's tumultuous adoption of free-market reforms after 1990 [49].

Nonetheless, there is some evidence that the overall nutritional quality of the Mongolian food supply has improved from 1990 to 2010 in terms of both increased per capita supply of healthy foods and nutrients, and decreased supply of unhealthy ones (more so than any other country) [50]. Shifts in specific dietary components during this period may have also predisposed Mongolians to weight loss and other positive metabolic changes independent of changes in caloric intake [47]; these include a 69.4 to $52.6 \mathrm{~g} /$ day decrease in intake of sugar-sweetened beverages and a 12.9 to $25.9 \mathrm{~g} /$ day increase in whole grains [40]. Despite these improvements, the annual reduction in age-standardized cardiovascular, type 2 diabetes, and cancer mortality attributable to dietary imbalances from 1990 to 2010 has been minimal $(-0.5 \%$, which is close to the median change observed in countries globally), while the magnitude of reductions in under-5 DALYs attributable to wasting $(-8.9 \%)$, stunting $(-10.6 \%)$, protein-energy malnutrition $(-11.6 \%)$, and deficiencies of iron $(-7.8 \%)$, zinc $(-11.0 \%)$, and other nutrients $(-11.5 \%)$ during this period has each been within the top $15 \%$ of countries [6]. This is in part attributable to the fact that national indicators of undernutrition respond relatively sensitively to increased food and nutrient availability, while NCDs do not on account of their longer longer latency periods. It can therefore be expected that ongoing improvements in the Mongolian food supply will have a more positive impact on NCD risk in the future, and this is borne out by current models that project a substantially increased annual reduction $(-2.1 \%)$ in age-standardized diet-related mortality from 2020 to 2040 [6].

To our knowledge, this has been the most detailed and expansive study of the diet of Mongolian adults to date. An important strength of this study was the use of paired sampling in summer and winter, which ensured that seasonal differences were largely un-confounded by participant characteristics. One limitation of the study was the fact that data were collected in only two seasons, although this limitation was mitigated by sampling during the peaks of summer and winter (which represent seasonal extremes in the Mongolian food supply). A second limitation was the lack of data on physical activity, which likely plays a role in differences in clinical measurements observed between subgroups, particularly urban-rural differences. To reduce bias in associational analyses, we attempted to stratify by or control for factors that may in part reflect physical activity (such as urbanicity, energy intake, or BMI), however, residual confounding is possible. Third, due to resource limitations, each year of the study involved data collection from up to three provinces only, and in each province, collection was completed over the course of one year; it was therefore not possible to examine changes in diet or nutrition that may have occurred in diet and nutrition from 2011 to 
2016 (which are possible, given rapid trends in urbanization and global market integration that have occurred in Mongolia in recent years) because such analyses would be intractably confounded by region. Fourth, despite the size and breadth of the study population (comprising 1838 person-days of summer and winter prospective dietary intake from urban and rural men and women in 8 national provinces), and the use of random sampling within each province, the study population is not intended to be nationally-representative and results should be interpreted in the context of the sampling frame. Readers of our earlier fortification modeling study [16], which includes national estimates for the baseline prevalence of dietary inadequacy of ten nutrients using data from the same population as the present study, will note moderate quantitative differences between those and the present results for thiamin and vitamin A in some subgroups. These differences may be attributable to differing methodologies used in estimating intake distributions as well as our earlier use of survey weights. The latter were necessary given the objective of that study to estimate and evaluate operational parameters of a national fortification program; for the explicit purpose of describing population thiamin and vitamin A adequacy, we thus suggest treating the estimates of both studies as realistic bounds of the true values.

\section{Conclusions}

Key dietary inadequacies and overweight are highly prevalent among Mongolian adults. Long-term strategies for addressing micronutrient deficits in Mongolia will require immense investment in agricultural infrastructure, trade and procurement policies, and demand generation designed to compensate for the country's environmental constraints that have historically limited fruit and vegetable availability. Similarly, Mongolia's NCD strategy requires development and calls for a multi-pronged approach, but would be cost-effective [51,52]. While contingent on efforts to increase the diversity and quality of foods supplied, one important strategy that will improve both micronutrient intake and NCD risk in Mongolia will be the revision of the country's dietary guidelines, which are both inadequately publicized and are not well-informed by nutrition science [53]. Finally, research priorities going forward should include the compilation, dissemination, and periodic update of national food composition data as many unique local dairy products and other foods cannot be easily imputed using foreign data; development, validation, and dissemination of standardized dietary assessment instruments; implementation of a national dietary surveillance system (potentially co-opting available household and commercial data platforms [54]); and continued development and follow-up of cohorts and other study populations for the purpose of nutritional epidemiology [55].

Supplementary Materials: The following are available online at http://www.mdpi.com/2072-6643/12/5/1514/s1, Table S1: Percentage of daily diet records in which food groups and alcohol were consumed, by subgroup-season and province; Table S2: Sample size of unique participants, daily diet records, and serum samples available for analysis by urbanicity, sex, season, and province; Table S3: Age distribution of study participants by urbanicity-sex and province; Table S4: Mean usual intake (g/day) of food groups by age within subgroup-seasons and Ulaanbaatar; Table S5: Prevalence of nutrient intakes above the upper limit by age, subgroup-season, and province.; Table S6: Mean usual nutrient intake (per day) and prevalence of nutrient inadequacy ( $\%<$ estimated average requirement) by age within subgroup-seasons and Ulaanbaatar; Table S7: Percentage of dietary nutrients contributed by consumed dishes by subgroup-season and province.

Author Contributions: Conceptualization, S.B., L.A.H., R.S.G., and D.G.; methodology, S.B., T.D., R.L.L., L.A.H., R.S.G., and D.G.; software, S.B.; formal analysis, S.B.; investigation, S.B., T.D., and D.G.; resources, R.L.L., S.T., and E.T.; data curation, S.B. and T.D.; writing-Original draft preparation, S.B. and R.S.G.; writing-Review and editing, T.D., R.L.L., S.T., L.A.H., E.T., N.G., and D.G.; visualization, S.B.; supervision, R.L.L., S.T., E.T., and N.G.; project administration, S.B., T.D., and D.G.; funding acquisition, S.B., L.A., R.S.G., and D.G. All authors have read and agreed to the published version of the manuscript.

Funding: This research received funding from the Harvard T.H. Chan School of Public Health Nutrition Round Table, Millennium Challenge Corporation, Sight and Life, Vitamin D Society, and the World Health Organization. SB was supported by the National Institute of Environmental Health Sciences (grant number: T32ES007069).

Acknowledgments: The authors thank Nachin Baasanjav for procuring support of research assistants and local health officers, and Jürgen Erhardt for contributing analysis of serum samples. 
Conflicts of Interest: The authors declare no conflict of interest. The funders had no role in the design of the study; in the collection, analyses, or interpretation of data; in the writing of the manuscript, or in the decision to publish the results.

\section{References}

1. World Bank. World Bank Open Data. Available online: https://data.worldbank.org (accessed on 2 February 2020).

2. Food and Agriculture Organization of the United Nations. FAOSTAT3 System. Available online: http: //faostat3.fao (accessed on 2 February 2020).

3. FAO; UNICEF; UNDP. Joint Food Security Assessment Mission to Mongolia; Food and Agriculture Organization of the United Nations: Ulaanbaatar, Mongolia, 2007.

4. Wang, D.D.; Li, Y.; Afshin, A.; Springmann, M.; Mozaffarian, D.; Stampfer, M.J.; Hu, F.B.; Murray, C.J.L.; Willett, W.C. Global Improvement in Dietary Quality Could Lead to Substantial Reduction in Premature Death. J. Nutr. 2019, 149, 1065-1074. [CrossRef]

5. Institute for Health Metrics and Evaluation. Global Burden of Disease Study 2017 (GBD 2017) Data Input Sources Tool. Available online: http:/ghdx.healthdata.org/gbd-2017/data-input-sources (accessed on 2 February 2020).

6. Institute for Health Metrics and Evaluation. GBD Compare. Available online: http://vizhub.healthdata.org/ gbd-compare (accessed on 2 February 2020).

7. Public Health Institute of Mongolia. Nutrition Status of the Population of Mongolia-5th National Nutrition Survey Report; Ministry of Health: Ulaanbaatar, Mongolia, 2017.

8. World Health Organization. Mongolian STEPS Survey on the Prevalence of Noncommunicable Disease and Injury Risk Factors-2013; World Health Organization: Ulaanbaatar, Mongolia, 2013.

9. Bromage, S.; Rich-Edwards, J.W.; Tselmen, D.; Baylin, A.; Houghton, L.A.; Baasanjav, N.; Ganmaa, D. Seasonal Epidemiology of Serum 25-Hydroxyvitamin D Concentrations among Healthy Adults Living in Rural and Urban Areas in Mongolia. Nutrients 2016, 8, 592. [CrossRef]

10. Ishii, S.; Samejima, K. Dietary survey on the diet of Mongolian nomads. J. Home Econ. Jpn. 1999, 50, 845-853.

11. Komatsu, F.; Hasegawa, K.; Watanabe, S.; Kawabata, T.; Yanagisawa, Y.; Kaneko, Y.; Miyagi, S.; Sakuma, M.; Kagawa, Y.; Ulziiburen, C.; et al. Comparison of electrocardiogram findings and lifestyles between urbanized people and ger-living people in Ulaanbaatar, Mongolia. Atherosclerosis 2004, 175, 101-108. [CrossRef]

12. Komatsu, F.; Kagawa, Y.; Sakuma, M.; Kawabata, T.; Kaneko, Y.; Otgontuya, D.; Chimedregzen, U.; Narantuya, L.; Purvee, B. Investigation of oxidative stress and dietary habits in Mongolian people, compared to Japanese people. Nutr. Metab. 2006, 3, 21. [CrossRef]

13. Komatsu, F.; Kagawa, Y.; Kawabata, T.; Kaneko, Y.; Purvee, B.; Otgon, J.; Chimedregzen, U. Dietary habits of Mongolian people, and their influence on lifestyle-related diseases and early aging. Curr. Aging Sci. 2008, 1, 84-100. [CrossRef]

14. Komatsu, F.; Kagawa, Y.; Kawabata, T.; Kaneko, Y.; Ishiguro, K. Relationship of dietary habits and obesity to oxidative stress in Palauan people: Compared with Japanese and Mongolian people. Curr. Aging Sci. 2009, 2, 214-222. [CrossRef]

15. Ohno, Y.; Hirai, K.; Sowa, S.; Oka, S.; Murai, Y. Food and nutrient intakes among nomads living in three different areas of Inner Mongolia, China. Asia Pac. J. Clin. Nutr. 2005, 14, 7-18.

16. Bromage, S.; Ganmaa, D.; Rich-Edwards, J.W.; Rosner, B.; Bater, J.; Fawzi, W.W. Projected effectiveness of mandatory industrial fortification of wheat flour, milk, and edible oil with multiple micronutrients among Mongolian adults. PLoS ONE 2018, 13, e0201230. [CrossRef]

17. Bromage, S.; Chung, H.Y.; Bonville, H.; Sprenger, J.C.; Lander, R.; Ganmaa, D. Trained Cohorts of University Students are a Useful Resource for Conducting Dietary Surveys in Mongolia. Food Nutr. Bull. 2017, 38, 267-272. [CrossRef]

18. WHO Expert Committee on Physical Status. Physical Status: The Use of and Interpretation of Anthropometry; Report of a WHO Expert Committee; World Health Organization: Geneva, Switzerland, 1995.

19. Shiwaku, K.; Anuurad, E.; Enkhmaa, B.; Nogi, A.; Kitajima, K.; Shimono, K.; Yamane, Y.; Oyunsuren, T. Overweight Japanese with body mass indexes of 23.0-24.9 have higher risks for obesity-associated disorders: A comparison of Japanese and Mongolians. Int. J. Obes. Relat. Metab. Disord. 2004, 28, 152-158. [CrossRef] 
20. Erhardt, J.G.; Estes, J.E.; Pfeiffer, C.M.; Biesalski, H.K.; Craft, N.E. Combined measurement of ferritin, soluble transferrin receptor, retinol binding protein, and C-reactive protein by an inexpensive, sensitive, and simple sandwich enzyme-linked immunosorbent assay technique. J. Nutr. 2004, 134, 3127-3132. [CrossRef]

21. Thurnham, D.I.; McCabe, G.P. Influence of infection and inflammation on biomarkers of nutritional status with an emphasis on vitamin A and iron. In Report: Priorities in the Assessment of Vitamin A and Iron Status in Populations, Panama City, Panama, 15-17 September 2010; World Health Organization: Geneva, Switzerland, 2012; pp. 63-80.

22. Adams, P. Management of elevated serum ferritin levels. Gastroenterol. Hepatol. 2008, 4, 333-334.

23. Food and Agriculture Organization of the United Nations. FAO/INFOODS e-Learning Course on Food Composition Data. Available online: http://www.fao.org/elearning/\#/elc/en/Course/FCD (accessed on 2 February 2020).

24. Bognár, A. Tables on Weight Yield of Food and Retention Factors of Food Constituents for the Calculation of Nutrient Composition of Cooked Foods (Dishes); Federal Research Centre for Nutrition and Food: Karlsruhe, Germany, 2002.

25. Matthews, R.H.; Garrison, Y.J. Agriculture Handbook No. 102: Food Yields Summarized by Different Stages of Preparation; USDA Agricultural Research Service: Washington, DC, USA, 1975.

26. Hartmann, B.M.; Bell, S.; Vásquez-Caicedo, A.L. Bundeslebensmittelschlüssel II 3.1; Federal Research Centre for Nutrition and Food: Karlsruhe, Germany, 2005.

27. Haytowitz, D.B. USDA National Nutrient Database for Standard Reference, Release 24; United States Department of Agriculture: Washington, DC, USA, 2011.

28. Lander, R.; Enkhjargal, T.S.; Batjargal, J.; Bolormaa, N.; Enkhmyagmar, D.; Tserendolgor, U.; Tungalag, S.; Bailey, K.; Gibson, R.S. Poor dietary quality of complementary foods is associated with multiple micronutrient deficiencies during early childhood in Mongolia. Public Health Nutr. 2010, 13, 1304-1313. [CrossRef]

29. Nutrient Data Laboratory. USDA Table of Nutrient Retention Factors; United States Department of Agriculture Agricultural Research Service: Beltsville, MD, USA, 2007.

30. Showell, B.A.; Williams, J.R.; Duvall, M.; Howe, J.C.; Patterson, K.Y.; Roseland, J.M.; Holden, J.M. USDA Table of Cooking Yields for Meat and Poultry; United States Department of Agriculture: Baltimore, MD, USA, 2012.

31. Dekkers, A.L.; Verkaik-Kloosterman, J.; van Rossum, C.T.; Ocké, M.C. SPADE, a new statistical program to estimate habitual dietary intake from multiple food sources and dietary supplements. J. Nutr. 2014, 144, 2083-2091. [CrossRef]

32. Institute of Medicine (US) Subcommittee on Interpretation and Uses of Dietary Reference Intakes; Institute of Medicine (US) Standing Committee on the Scientific Evaluation of Dietary Reference Intakes. DRI Dietary Reference Intakes: Applications in Dietary Planning; National Academies Press: Washington, DC, USA, 2000.

33. National Academy of Medicine. DRI Summary Tables. Available online: https://www.nal.usda.gov/sites/ default/files/fnic_uploads//SummaryTables.pdf (accessed on 2 February 2020).

34. Lander, R.L.; Enkhjargal, T.; Batjargal, J.; Bailey, K.B.; Diouf, S.; Green, T.J.; Skeaff, C.M.; Gibson, R.S. Multiple micronutrient deficiencies persist during early childhood in Mongolia. Asia Pac. J. Clin. Nutr. 2008, 17, 429-440.

35. Baatar, N.; Enkhbat, A.; Shagdarsuren, U.; Baigali, D.; Shuumarjav, U.; Malchinkhuu, M.; Nansalmaa, E.; Tserenkhuu, L.; Enkhtaivan, O. Osteoporosis in Mongolian Population: Prevalence and Risk Factors. Cent. Asian J. Med. Sci. 2017, 3, 282-288.

36. Pereira, M.A.; O’Reilly, E.; Augustsson, K.; Fraser, G.E.; Goldbourt, U.; Heitmann, B.L.; Hallmans, G.; Knekt, P.; Liu, S.; Pietinen, P.; et al. Dietary fiber and risk of coronary heart disease: A pooled analysis of cohort studies. Arch. Intern. Med. 2004, 164, 370-376. [CrossRef]

37. McKeown, N.M.; Meigs, J.B.; Liu, S.; Saltzman, E.; Wilson, P.W.; Jacques, P.F. Carbohydrate nutrition, insulin resistance, and the prevalence of the metabolic syndrome in the Framingham Offspring Cohort. Diabetes Care 2004, 27, 538-546. [CrossRef]

38. Schulze, M.B.; Liu, S.; Rimm, E.B.; Manson, J.E.; Willett, W.C.; Hu, F.B. Glycemic index, glycemic load, and dietary fiber intake and incidence of type 2 diabetes in younger and middle-aged women. Am. J. Clin. Nutr. 2004, 80, 348-356. [CrossRef]

39. Enkhmaa, D.; Tanz, L.; Ganmaa, D.; Enkhtur, S.; Oyun-Erdene, B.; Stuart, J.; Chen, G.; Carr, A.; Seely, E.W.; Fitzmaurice, G.; et al. Randomized trial of three doses of vitamin D to reduce deficiency in pregnant Mongolian women. EBioMedicine 2019, 39, 510-519. [CrossRef] [PubMed] 
40. Khatibzadeh, S.; Saheb-Kashaf, M.; Micha, R.; Fahimi, S.; Shi, P.; Elmadfa, I.; Kalantarian, S.; Wirojratana, P.; Ezzati, M.; Powles, J.; et al. Global Burden of Diseases Nutrition; Chronic Diseases Expert Group (NutriCode). A global database of food and nutrient consumption. Bull. World Health Organ. 2016, 94, 931-934. [CrossRef] [PubMed]

41. Orsavova, J.; Misurcova, L.; Ambrozova, J.V.; Vicha, R.; Mlcek, J. Fatty Acids Composition of Vegetable Oils and Its Contribution to Dietary Energy Intake and Dependence of Cardiovascular Mortality on Dietary Intake of Fatty Acids. Int. J. Mol. Sci. 2015, 16, 12871-12890. [CrossRef]

42. Mozaffarian, D.; Micha, R.; Wallace, S. Effects on coronary heart disease of increasing polyunsaturated fat in place of saturated fat: A systematic review and meta-analysis of randomized controlled trials. PLoS Med. 2010, 7, e1000252. [CrossRef]

43. Mensink, R.P.; Zock, P.L.; Kester, A.D.; Katan, M.B. Effects of dietary fatty acids and carbohydrates on the ratio of serum total to HDL cholesterol and on serum lipids and apolipoproteins: A meta-analysis of 60 controlled trials. Am. J. Clin. Nutr. 2003, 77, 1146-1155. [CrossRef]

44. Appel, L.J.; Sacks, F.M.; Carey, V.J.; Obarzanek, E.; Swain, J.F.; Miller, E.R., 3rd; Conlin, P.R.; Erlinger, T.P.; Rosner, B.A.; Laranjo, N.M.; et al. Effects of protein, monounsaturated fat, and carbohydrate intake on blood pressure and serum lipids: Results of the OmniHeart randomized trial. JAMA 2005, 294, 2455-2464. [CrossRef]

45. Riserus, U.; Willett, W.C.; Hu, F.B. Dietary fats and prevention of type 2 diabetes. Prog. Lipid Res. 2009, 48, 44-51. [CrossRef]

46. Dugee, O.; Khor, G.L.; Lye, M.S.; Luvsannyam, L.; Janchiv, O.; Jamyan, B.; Esa, N. Association of major dietary patterns with obesity risk among Mongolian men and women. Asia Pac. J. Clin. Nutr. 2009, 18, 433-440.

47. Mozaffarian, D.; Hao, T.; Rimm, E.B.; Willett, W.C.; Hu, F.B. Changes in diet and lifestyle and long-term weight gain in women and men. N. Engl. J. Med. 2011, 364, 2392-2404. [CrossRef]

48. GBD 2015 Obesity Collaborators. Health Effects of Overweight and Obesity in 195 Countries over 25 Years. N. Engl. J. Med. 2017, 377, 13-27. [CrossRef]

49. McMurray, C.; Smith, R. Diseases of Globalization, Socioeconomic Transitions and Health; Earthscan: London, UK, 2001.

50. Imamura, F.; Micha, R.; Khatibzadeh, S.; Fahimi, S.; Shi, P.; Powles, J.; Mozaffarian, D.; Global Burden of Diseases Nutrition and Chronic Diseases Expert Group (NutriCoDE). Dietary quality among men and women in 187 countries in 1990 and 2010: A systematic assessment. Lancet Glob. Health 2015, 3, e132-e142. [CrossRef]

51. Chimeddamba, O.; Peeters, A.; Walls, H.L.; Joyce, C. Noncommunicable Disease Prevention and Control in Mongolia: A Policy Analysis. BMC Public Health 2015, 15, 660. [CrossRef] [PubMed]

52. World Health Organization; United Nations Development Programme. The Investment Case for Noncommunicable Disease Prevention and Control in Mongolia: Return on Investment Analysis and Institutional Context Analysis. Available online: https://apps.who.int/iris/handle/10665/259627 (accessed on 2 February 2020).

53. Food and Agriculture Organization of the United Nations. Food-Based Dietary Guidelines-Mongolia. Available online: www.fao.org/nutrition/education/food-dietary-guidelines/regions/countries/mongolia/en (accessed on 2 February 2020).

54. Bromage, S.; Rosner, B.; Rich-Edwards, J.W.; Ganmaa, D.; Tsolmon, S.; Tserendejid, Z.; Odbayar, T.O.; Traeger, M.; Fawzi, W.W. Comparison of Methods for Estimating Dietary Food and Nutrient Intakes and Intake Densities from Household Consumption and Expenditure Data in Mongolia. Nutrients 2018, 10, 703. [CrossRef] [PubMed]

55. Enkh-Oyun, T.; Davaalkham, D.; Kotani, K.; Aoyama, Y.; Tsuboi, S.; Ae, R.; Davaa, G.; Angarmurun, D.; Khuderchuluun, N.; Nakamura, Y. A cohort study of chronic diseases for Mongolian people: Outline with baseline data of the Moncohort study. J. Epidemiol. Glob. Health 2016, 6, 187-196. [CrossRef] [PubMed]

(C) 2020 by the authors. Licensee MDPI, Basel, Switzerland. This article is an open access article distributed under the terms and conditions of the Creative Commons Attribution (CC BY) license (http://creativecommons.org/licenses/by/4.0/). 